\title{
Boundary value problems for a special Helfrich functional for surfaces of revolution: existence and asymptotic behaviour
}

\author{
Klaus Deckelnick ${ }^{1} \cdot$ Marco Doemeland ${ }^{2} \cdot$ Hans-Christoph Grunau ${ }^{1}$
}

Received: 23 March 2020 / Accepted: 30 September 2020 / Published online: 18 January 2021

(c) The Author(s) 2021

\begin{abstract}
The central object of this article is (a special version of) the Helfrich functional which is the sum of the Willmore functional and the area functional times a weight factor $\varepsilon \geq 0$. We collect several results concerning the existence of solutions to a Dirichlet boundary value problem for Helfrich surfaces of revolution and cover some specific regimes of boundary conditions and weight factors $\varepsilon \geq 0$. These results are obtained with the help of different techniques like an energy method, gluing techniques and the use of the implicit function theorem close to Helfrich cylinders. In particular, concerning the regime of boundary values, where a catenoid exists as a global minimiser of the area functional, existence of minimisers of the Helfrich functional is established for all weight factors $\varepsilon \geq 0$. For the singular limit of weight factors $\varepsilon \nearrow \infty$ they converge uniformly to the catenoid which minimises the surface area in the class of surfaces of revolution.
\end{abstract}

\section{Introduction}

The Helfrich energy for a sufficiently smooth (two dimensional) surface $S \subset \mathbb{R}^{3}$ (with or without boundary), introduced by Helfrich in [27] and Canham in [8], is defined by

$$
\mathscr{H}(S):=\int_{S}\left(H-H_{0}\right)^{2} \mathrm{~d} S-\gamma \int_{S} K \mathrm{~d} S+\varepsilon \int_{S} \mathrm{~d} S .
$$

Here the integration is done with respect to the usual 2-dimensional area measure $\mathrm{d} S, H$ is the mean curvature of $S$, i. e. the mean value of the principal curvatures, $K$ is the Gaussian curvature, $\gamma \in \mathbb{R}$ is a constant bending rigidity, $\varepsilon \geq 0$ the weight factor of the area functional and $H_{0} \in \mathbb{R}$ a given spontaneous curvature. For simplicity we will always assume $H_{0}=0$ so that the first term in the above energy reduces to the well-known Willmore functional $\int_{S} H^{2} \mathrm{~d} S$. We expect nontrivial $H_{0} \neq 0$ to result in completely different geometric phenom-

Communicated by A.Malchiodi.

Marco Doemeland

doemeland@eddy.rwth-aachen.de

1 Fakultät für Mathematik, Otto-von-Guericke-Universität Magdeburg, Postfach 4120, 39016 Magdeburg, Germany

2 Math 111810, RWTH Aachen University, 52056 Aachen, Germany 
ena; we think that our methods cannot directly be extended to this case. For the investigation of closed surfaces in the case $H_{0} \neq 0$ one may see e.g. [5,10]. We also choose $\gamma=0$ since $\int_{S} K \mathrm{~d} S$ in the second term will only contribute to boundary terms by the Gauss-Bonnet theorem, which are constant thanks to the given boundary conditions. The Euler-Lagrange equation to the resulting Helfrich energy is known as the Helfrich equation and is given by

$$
\Delta_{S} H+2 H\left(H^{2}-K\right)-2 \varepsilon H=0 \quad \text { on } S,
$$

where $\Delta_{S}$ denotes the Laplace-Beltrami operator on $S$.

There are several applications of the Helfrich energy in e.g. biology in modelling red blood cells or lipid bilayers (see e.g. $[8,27,34,36]$ ) and in modelling thin elastic plates (see e. g. [24]). As noted in [11,34], the Willmore functional was already considered in the early 19 th century (see $[24,37]$ ) and has been periodically reintroduced according to the availability of more powerful mathematical tools e.g. in the early 1920s (see [45]) and most successfully by Willmore (see [46]) in the 1960s. So far, mathematical research has mainly focused on closed (i.e. compact without boundary) surfaces minimising the Willmore functional, see e.g. $[2,32,44]$ and references therein. Some results on closed surfaces minimising the Helfrich energy under fixed area and enclosed volume for the axisymmetric case can be found in [10]. In [9] this was generalised to a multiphase Helfrich energy, while recently in [6] the same problem was studied in the setting of curvature varifolds, thereby dropping the symmetry assumptions.

In contrast to closed surfaces we are here interested in a Dirichlet boundary value problem for minimising the Helfrich energy. Some existence results on the Dirichlet problem for the Willmore functional can be found e.g. in [42] for a class of branched immersions in $\mathbb{R}^{3} \cup\{\infty\}$. The Douglas (or Navier) boundary value problem was studied in [35,38]. In the class of surfaces of revolution existence results for the Willmore functional were obtained in $[12,14,20]$; see also references therein. In [16] existence of minimisers of a relaxed Willmore functional in the class of graphs over two-dimensional domains was proved. For the Helfrich functional an existence result for branched immersions in $\mathbb{R}^{3}$ was found in [19]. This is somehow related to [11], where an area constraint was imposed in order to minimise the Willmore functional.

In this paper we focus on surfaces of revolution $S$

$$
(x, \theta) \mapsto(x, u(x) \cos \theta, u(x) \sin \theta), \quad x \in[-1,1], \theta \in[0,2 \pi],
$$

for some sufficiently smooth profile curve $u:[-1,1] \rightarrow(0, \infty)$. We then consider the Dirichlet boundary value problem for Helfrich surfaces of revolution

$$
\left\{\begin{array}{l}
\Delta_{S} H+2 H\left(H^{2}-K\right)-2 \varepsilon H=0 \quad \text { in }(-1,1), \\
u( \pm 1)=\alpha, \quad u^{\prime}( \pm 1)=0,
\end{array}\right.
$$

for a given boundary value $\alpha>0$ and for profile curves $u$ that are even, i. e. $u(x)=u(-x)$. The precise setting and a derivation of (1) will be given in Sect. 2. First existence results for (1) have been obtained in [43] and [18]. The goal of this paper is to extend these results in several directions including a description of the qualitative behaviour of solutions when possible. Depending on the two parameters $\alpha$ and $\varepsilon$, Fig. 1 gives an overview of domains on which existence of solutions to the Helfrich problem (1) is guaranteed. Let us describe these domains in more detail and relate them to the various sections of this paper.

As a starting point we consider in Sect. 3 the Helfrich boundary value problem as a regular perturbation of the Willmore boundary value problem for small $\varepsilon$. Note however that a direct application of the techniques developed in [12] for the Willmore functional is not possible 


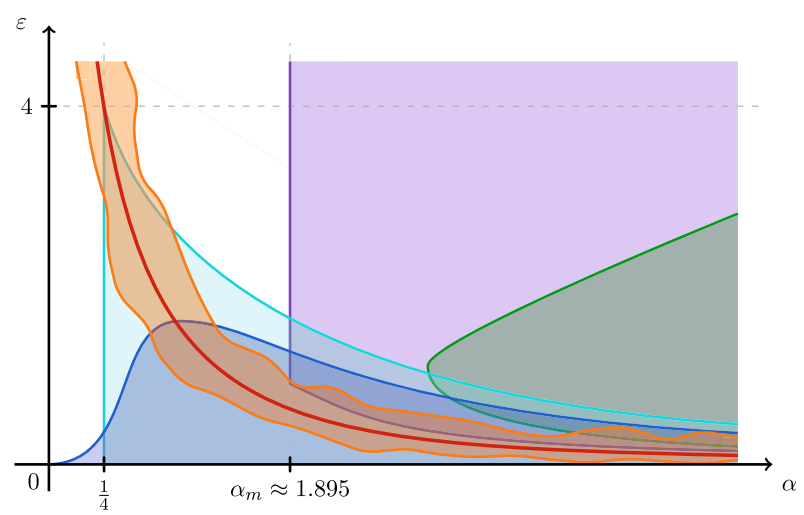

Fig. 1 A sketch of domains for existence of solutions to the Dirichlet problem (1) depending on $(\alpha, \varepsilon)$ : The domains blue and light blue show existence of Helfrich minimisers obtained in Theorem 2 and [18, Satz 5.3.3 and 5.3.4]. The red curve where $\varepsilon=\varepsilon_{\alpha}=\frac{1}{4 \alpha^{2}}$ displays the Helfrich cylinders (see [43, Lemma 4.1]). The orange domain sketches existence of solutions obtained by perturbing the Helfrich cylinders (see Theorem 3). The purple domain is obtained with the help of gluing techniques (see Theorem 7) and may be compared to the green domain implicitly found in [43, Theorem 4.14] (colour figure online)

because they are heavily based on its conformal invariance and on explicitly known Willmore minimisers. Nevertheless, by applying the direct method and utilising an energy gap of the Willmore energy to $4 \pi$ we find in Theorem 2 solutions of (1) for any $\alpha>0$ and $\varepsilon \geq 0$ not too large, see the blue regions in Fig. 1. In this case we solely give a quantitative existence proof with no qualitative description of the minimisers.

In order to develop some intuition for what happens when $\varepsilon$ increases we then concentrate in Sect. 4 on the local picture around a Helfrich cylinder, i.e. $u(x) \equiv \alpha$, which is an explicit solution and the unique Helfrich minimiser in the case $\varepsilon=\varepsilon_{\alpha}:=\frac{1}{4 \alpha^{2}}$ (see [43, Lemma 4.1]; red curve in Fig. 1). Linearising (1) for fixed $\alpha$ at the corresponding Helfrich cylinder, the implicit function theorem yields the existence of a smooth family $\left(u_{\varepsilon}\right)_{\varepsilon \approx \varepsilon_{\alpha}}$ of solutions of (1), see Theorem 3; orange domain in Fig. 1. As a first step in order to understand whether and how this local branch is part of a possibly existing family of solutions $\left(u_{\varepsilon}\right)_{\varepsilon \geq 0}$ we consider the rate of change function $r c_{\alpha}:=\left.\frac{\partial u_{\varepsilon}}{\partial \varepsilon}\right|_{\varepsilon=\varepsilon_{\alpha}}$, for which we derive an explicit formula. A careful analysis of the resulting expression shows that $r c_{\alpha}$ is negative, see Theorem 4 , which gives rise to the (open) conjecture that the family $\left(u_{\varepsilon}\right)_{\varepsilon \in\left[0, \varepsilon_{\alpha}\right]}$ exists and is decreasing with respect to $\varepsilon$. Furthermore, we find that the shape of $r c_{\alpha}$ strongly depends on the boundary value $\alpha$. More precisely, there exists a critical value $\alpha_{\text {crit }} \approx 0.18008$ such that for $\alpha>\alpha_{\text {crit }}$ the function $r c_{\alpha}$ is strictly increasing on [0,1] (see Theorem 5), while for $\alpha<\alpha_{\text {crit }}$ and $\alpha \searrow 0$ an increasing number of oscillations around a negative value show up. This interesting phenomenon of "overshoot" and subsequent oscillations around an expected "attracting state" has often been observed in numerical experiments and to our knowledge this is the first time where an analytical proof is given. It indicates that for small $\alpha>0$, understanding existence, qualitative properties and asymptotic behaviour for the full range of $\varepsilon \in[0, \infty)$ remains an interesting and challenging open problem.

Next, in Sect. 5 we consider the case of larger values of $\varepsilon$. This means that the area functional as part of the Helfrich energy is given a bigger weight suggesting that minimisers of the area functional in the class of surfaces of revolution might be useful when minimising the Helfrich funtional in this case. It is well-known that there exists a threshold $\alpha_{m} \approx 1.805$ 
such that for $\alpha>\alpha_{m}$ the minimiser is given by a catenoid while for $\alpha<\alpha_{m}$ it is a so-called Goldschmidt solution. Using this observation in the case $\alpha \geq \alpha_{m}$ we are able to decrease the energy of minimising sequences for the Helfrich functional by gluing in suitable catenoids leading to the existence of minimisers, see Theorem 7 and the purple region in Fig. 1. At the same time this process gives some qualitative information about these minimisers. In addition, we prove in Corollary 3 that the sequence of minimisers converges to the areaminimising catenoid in the singular limit $\varepsilon \nearrow \infty$. So far, there is only numerical evidence that for $0<\alpha<\alpha_{m}$ minimisers may exist for any $\varepsilon \geq 0$ and converge in some singular sense to the Goldschmidt minimal surface as $\varepsilon \nearrow \infty$.

In Sect. 6 we finally summarise our results and describe some open problems. At the end we add three appendices. Appendix A provides a proof for the "well-known" fact, which catenoid minimises the area functional. Appendix B proves a divergence form of the Helfrich equation and Appendix C collects the basic estimates to prove the theorems of Sect. 4.

\section{Geometric background for surfaces of revolution}

For a sufficiently smooth (two dimensional) surface $S \subset \mathbb{R}^{3}$ (with or without boundary) we consider the Helfrich functional (see e.g. [27,34])

$$
\mathscr{H}(S):=\int_{S} H^{2} \mathrm{~d} S-\gamma \int_{S} K \mathrm{~d} S+\varepsilon \int_{S} \mathrm{~d} S .
$$

Here $\mathrm{d} S$ denotes integration with respect to the surface area measure, while

$$
H(x):=\frac{1}{2}\left(\kappa_{1}(x)+\kappa_{2}(x)\right) \text { and } \quad K(x):=\kappa_{1}(x) \kappa_{2}(x)
$$

are the mean curvature and the Gauss curvature of $S$ respectively. Furthermore, $\gamma \in \mathbb{R}$ as well as $\varepsilon \geq 0$ are given constants. In what follows we consider surfaces of revolution $S$

$$
(x, \theta) \mapsto(x, u(x) \cos \theta, u(x) \sin \theta), \quad x \in[-1,1], \theta \in[0,2 \pi],
$$

which arise when the sufficiently smooth profile curve $u:[-1,1] \rightarrow(0, \infty)$ is rotated around the $x$-axis. Expressing $H$ and $K$ in terms of $u$ yields

$$
\begin{aligned}
& H(x)=\frac{1}{2}\left(\frac{1}{u(x) \sqrt{1+u^{\prime}(x)^{2}}}-\frac{u^{\prime \prime}(x)}{\left(1+u^{\prime}(x)^{2}\right)^{3 / 2}}\right), \\
& K(x)=-\frac{u^{\prime \prime}(x)}{u(x)\left(1+u^{\prime}(x)^{2}\right)^{2}} .
\end{aligned}
$$

Since $\mathrm{d} S=u(x) \sqrt{1+u^{\prime}(x)^{2}} \mathrm{~d} x \mathrm{~d} \theta$ we have for the surface area

$$
\mathscr{A}(u):=2 \pi \int_{-1}^{1} u(x) \sqrt{1+u^{\prime}(x)^{2}} \mathrm{~d} x
$$

while the Willmore functional is given by

$$
\mathscr{W}(u):=\frac{\pi}{2} \int_{-1}^{1}\left(\frac{1}{u(x) \sqrt{1+u^{\prime}(x)^{2}}}-\frac{u^{\prime \prime}(x)}{\left(1+u^{\prime}(x)^{2}\right)^{3 / 2}}\right)^{2} u(x) \sqrt{1+u^{\prime}(x)^{2}} \mathrm{~d} x .
$$

In what follows we shall consider for $\alpha>0$ the following class of admissible functions:

$$
N_{\alpha}:=\left\{u \in H^{2}(-1,1): u \text { is even, } u>0 \text { in }[-1,1], u( \pm 1)=\alpha, u^{\prime}( \pm 1)=0\right\} .
$$


Note that for $u \in N_{\alpha}$

$$
\int_{S} K \mathrm{~d} S=-2 \pi \int_{-1}^{1} \frac{u^{\prime \prime}(x)}{\left(1+u^{\prime}(x)^{2}\right)^{3 / 2}} \mathrm{~d} x=-2 \pi\left[\frac{u^{\prime}(x)}{\sqrt{1+u^{\prime}(x)^{2}}}\right]_{x=-1}^{x=1}=0,
$$

so that in our setting the Helfrich functional $\mathscr{H}=\mathscr{H}_{\varepsilon}: N_{\alpha} \rightarrow \mathbb{R}$ takes the form

$$
\mathscr{H}_{\varepsilon}(u):=\mathscr{W}(u)+\varepsilon \mathscr{A}(u) .
$$

Using the fact that $\frac{u^{\prime \prime}}{\left(1+\left(u^{\prime}\right)^{2}\right)^{3 / 2}}=\frac{\mathrm{d}}{\mathrm{d} x}\left(\frac{u^{\prime}}{\sqrt{1+\left(u^{\prime}\right)^{2}}}\right)$ we find that

$$
\begin{aligned}
\int_{a}^{b} & \left(\frac{1}{u(x) \sqrt{1+u^{\prime}(x)^{2}}}-\frac{u^{\prime \prime}(x)}{\left(1+u^{\prime}(x)^{2}\right)^{3 / 2}}\right)^{2} u(x) \sqrt{1+u^{\prime}(x)^{2}} \mathrm{~d} x \\
= & \int_{a}^{b}\left[\left(\frac{1}{u(x) \sqrt{1+u^{\prime}(x)^{2}}}\right)^{2}+\left(\frac{u^{\prime \prime}(x)}{\left(1+u^{\prime}(x)^{2}\right)^{3 / 2}}\right)^{2}\right] u(x) \sqrt{1+u^{\prime}(x)^{2}} \mathrm{~d} x \\
& -\left.2 \frac{u^{\prime}(x)}{\sqrt{1+u^{\prime}(x)^{2}}}\right|_{a} ^{b} \\
= & \int_{a}^{b}\left(\frac{1}{u(x) \sqrt{1+u^{\prime}(x)^{2}}}+\frac{u^{\prime \prime}(x)}{\left(1+u^{\prime}(x)^{2}\right)^{3 / 2}}\right)^{2} u(x) \sqrt{1+u^{\prime}(x)^{2}} \mathrm{~d} x \\
& -\left.4 \frac{u^{\prime}(x)}{\sqrt{1+u^{\prime}(x)^{2}}}\right|_{a} ^{b} \cdot
\end{aligned}
$$

In particular we have for $u \in N_{\alpha}$ that

$$
\begin{aligned}
\mathscr{H}_{\varepsilon}(u)= & \frac{\pi}{2} \int_{-1}^{1}\left(\frac{1}{u(x) \sqrt{1+u^{\prime}(x)^{2}}}+4 \varepsilon u(x) \sqrt{1+u^{\prime}(x)^{2}}\right) \mathrm{d} x \\
& +\frac{\pi}{2} \int_{-1}^{1} \frac{u(x) u^{\prime \prime}(x)^{2}}{\left(1+u^{\prime}(x)^{2}\right)^{5 / 2}} \mathrm{~d} x .
\end{aligned}
$$

Let us next consider the Euler-Lagrange equation for $\mathscr{H}_{\varepsilon}$ and fix $u \in N_{\alpha} \cap C^{4}([-1,1])$. Then we calculate for the first variation of $\mathscr{A}$ at $u$ in direction $\varphi \in H_{0}^{2}(-1,1)$

$$
\begin{aligned}
\left\langle\mathscr{A}^{\prime}(u), \varphi\right\rangle & =2 \pi \int_{-1}^{1}\left[\varphi(x) \sqrt{1+u^{\prime}(x)^{2}}+\frac{u(x) u^{\prime}(x) \varphi^{\prime}(x)}{\sqrt{1+u^{\prime}(x)^{2}}}\right] \mathrm{d} x \\
& =4 \pi \int_{-1}^{1} u(x) \varphi(x) H(x) \mathrm{d} x
\end{aligned}
$$

while [15, Lemma A.1] yields

$$
\left\langle\mathscr{W}^{\prime}(u), \varphi\right\rangle=-2 \pi \int_{-1}^{1} u(x) \varphi(x)\left(\Delta_{S} H+2 H\left(H^{2}-K\right)\right)(x) \mathrm{d} x .
$$

Combining (9) and (10) we see that if $u \in N_{\alpha} \cap C^{4}([-1,1])$ is a critical point of the energy $\mathscr{H}_{\varepsilon}$ then it is a solution of Helfrich equation

$$
\Delta_{S} H+2 H\left(H^{2}-K\right)-2 \varepsilon H=0 \quad \text { in }(-1,1) .
$$


Using the calculations in [12, Section 2.1] in order to express the left hand side in terms of $u$ we obtain that $u$ is a solution of the following Dirichlet problem

$$
\begin{aligned}
& \frac{1}{u(x) \sqrt{1+u^{\prime}(x)^{2}}} \frac{\mathrm{d}}{\mathrm{d} x}\left(\frac{u(x)}{\sqrt{1+u^{\prime}(x)^{2}}} H^{\prime}(x)\right) \\
& +\frac{1}{2} H(x)\left(\frac{u^{\prime \prime}(x)}{\left(1+u^{\prime}(x)^{2}\right)^{3 / 2}}+\frac{1}{u(x) \sqrt{1+u^{\prime}(x)^{2}}}\right)^{2}-2 \varepsilon H(x)=0, \quad x \in(-1,1) \\
& u( \pm 1)=\alpha, u^{\prime}( \pm 1)=0 .
\end{aligned}
$$

Note that (12) is a fourth order quasilinear equation, which is elliptic, but not uniformly elliptic. Let us briefly refer to three particular solutions of (12) in the class of symmetric, positive profile curves:

i. catenoids: $u(x)=c \cosh \left(\frac{x}{c}\right)(c>0)$ for every $\varepsilon \geq 0$.

ii. spheres: $u(x)=\sqrt{c^{2}-x^{2}}(c>1)$ in the case $\varepsilon=0$;

iii. cylinders: $u(x)=c>0$ in the case $\varepsilon=\frac{1}{4 c^{2}}$.

Catenoids and spheres have been successfully employed in the construction of symmetric Willmore surfaces (see e.g. [12], [14]) as well as in the analysis of their asymptotic shape for small values of $\alpha$, see [26].

\section{Existence of minimisers via energy bounds}

Lemma 1 Let $u \in H^{2}(-1,1)$ be even.

a) If $u^{\prime}( \pm 1)=0$, then $\mathscr{A}(u) \mathscr{W}(u) \geq 4 \pi^{2}$.

b) If $\mathscr{W}(u)<4 \pi$ and $u^{\prime}( \pm 1)=0$, then

$$
\max _{x \in[-1,1]}\left|u^{\prime}(x)\right| \leq \frac{\mathscr{W}(u)}{\sqrt{16 \pi^{2}-\mathscr{W}(u)^{2}}} .
$$

c) If $\left|u^{\prime}(x)\right| \leq M, u( \pm 1)=\alpha$, then

$$
\forall x \in[-1,1]: \quad \alpha \exp \left(-\frac{1}{\pi} M \sqrt{1+M^{2}} \mathscr{W}(u)\right) \leq u(x) \leq \alpha+M .
$$

Proof a) Using Hölder's inequality and (7) we have

$$
\begin{aligned}
4 \pi & \leq 2 \pi \int_{-1}^{1} \underbrace{\sqrt{\left(\frac{1}{u(x) \sqrt{1+u^{\prime}(x)^{2}}}\right)^{2}+\underbrace{\left(\frac{u^{\prime \prime}(x)}{\left(1+u^{\prime}(x)^{2}\right)^{3 / 2}}\right)^{2}}} u(x) \sqrt{1+u^{\prime}(x)^{2}}}_{\geq 1} \mathrm{~d} x \\
& \leq \sqrt{2 \pi}\left(\int_{-1}^{1}\left[\left(\frac{1}{u(x) \sqrt{1+u^{\prime}(x)^{2}}}\right)^{2}+\left(\frac{u^{\prime \prime}(x)}{\left(1+u^{\prime}(x)^{2}\right)^{3 / 2}}\right)^{2}\right] u(x) \sqrt{1+u^{\prime}(x)^{2}} \mathrm{~d} x\right)^{\frac{1}{2}} \sqrt{\mathscr{A}(u)} \\
& =\sqrt{2 \pi}\left(\int_{-1}^{1}\left(\frac{1}{u(x) \sqrt{1+u^{\prime}(x)^{2}}}-\frac{u^{\prime \prime}(x)}{\left(1+u^{\prime}(x)^{2}\right)^{3 / 2}}\right)^{2} u(x) \sqrt{1+u^{\prime}(x)^{2}} \mathrm{~d} x\right)^{\frac{1}{2}} \sqrt{\mathscr{A}(u)} \\
& =2 \sqrt{\mathscr{W}(u)} \sqrt{\mathscr{A}(u) .}
\end{aligned}
$$


b) Choose $x_{0} \in(0,1)$ with $\left|u^{\prime}\left(x_{0}\right)\right|=\max _{x \in[-1,1]}\left|u^{\prime}(x)\right|$ and let $[a, b]=\left[x_{0}, 1\right]$ if $u^{\prime}\left(x_{0}\right) \geq$ 0 and $[a, b]=\left[0, x_{0}\right]$ if $u^{\prime}\left(x_{0}\right)<0$. Since $u^{\prime}(0)=u^{\prime}(1)=0$ we calculate with the help of (7)

$$
\begin{aligned}
\mathscr{W}(u) \geq & \pi \int_{a}^{b}\left(\frac{1}{u(x) \sqrt{1+u^{\prime}(x)^{2}}}-\frac{u^{\prime \prime}(x)}{\left(1+u^{\prime}(x)^{2}\right)^{3 / 2}}\right)^{2} u(x) \sqrt{1+u^{\prime}(x)^{2}} \mathrm{~d} x \\
= & \pi \int_{a}^{b}\left(\frac{1}{u(x) \sqrt{1+u^{\prime}(x)^{2}}}+\frac{u^{\prime \prime}(x)}{\left(1+u^{\prime}(x)^{2}\right)^{3 / 2}}\right)^{2} u(x) \sqrt{1+u^{\prime}(x)^{2}} \mathrm{~d} x \\
& -\left.4 \pi \frac{u^{\prime}(x)}{\sqrt{1+u^{\prime}(x)^{2}}}\right|_{a} ^{b} \\
\geq & 4 \pi \frac{\left|u^{\prime}\left(x_{0}\right)\right|}{\sqrt{1+u^{\prime}\left(x_{0}\right)^{2}}}
\end{aligned}
$$

and the required bound follows by solving for $\left|u^{\prime}\left(x_{0}\right)\right|$.

c) For $x \in[0,1]$ we have

$$
\begin{aligned}
\log u(x) & =\log u(1)-\int_{x}^{1} \frac{u^{\prime}(t)}{u(t)} \mathrm{d} t \geq \log \alpha-M \sqrt{1+M^{2}} \int_{0}^{1} \frac{1}{u(t) \sqrt{1+u^{\prime}(t)^{2}}} \mathrm{~d} t \\
& \geq \log \alpha-\frac{1}{\pi} M \sqrt{1+M^{2}} \mathscr{W}(u),
\end{aligned}
$$

which implies the lower bound on $u$. A similar idea was used in [14, Lemma 4.9]. The upper bound is immediate.

Corollary 1 Suppose that $u \in H^{2}(-1,1)$ is even with $u^{\prime}( \pm 1)=0$. Then $\mathscr{H}_{\varepsilon}(u) \geq 4 \pi \sqrt{\varepsilon}$ and

$$
\mathscr{W}(u) \leq \frac{1}{2}\left(\mathscr{H}_{\varepsilon}(u)+\sqrt{\mathscr{H}_{\varepsilon}(u)^{2}-16 \pi^{2} \varepsilon}\right) .
$$

Proof Lemma 1 a) implies that

$$
\mathscr{H}_{\varepsilon}(u)=\mathscr{W}(u)+\varepsilon \mathscr{A}(u) \geq \mathscr{W}(u)+\frac{4 \pi^{2} \varepsilon}{\mathscr{W}(u)}
$$

and hence

$$
\mathscr{W}(u)^{2}-\mathscr{H}_{\varepsilon}(u) \mathscr{W}(u)+4 \pi^{2} \varepsilon \leq 0,
$$

which yields (14).

Theorem 1 Let $\alpha>0$ and $0 \leq \varepsilon \leq 4$. If $\mathscr{H}_{\varepsilon}(v)<\pi(4+\varepsilon)$ for some $v \in N_{\alpha}$, then $\mathscr{H}_{\varepsilon}$ attains a minimum $u \in N_{\alpha}$, which belongs to $C^{\infty}([-1,1])$.

Proof Let $\left(u_{k}\right)_{k \in \mathbb{N}} \subset N_{\alpha}$ be a minimising sequence such that $\mathscr{H}_{\varepsilon}\left(u_{k}\right) \searrow \inf { }_{w \in N_{\alpha}} \mathscr{H}_{\varepsilon}(w) \leq$ $\mathscr{H}_{\varepsilon}(v)$. Corollary 1 implies that

$$
\begin{aligned}
\limsup _{k \rightarrow \infty} \mathscr{W}\left(u_{k}\right) & \leq \frac{1}{2} \lim _{k \rightarrow \infty}\left(\mathscr{H}_{\varepsilon}\left(u_{k}\right)+\sqrt{\mathscr{H}_{\varepsilon}\left(u_{k}\right)^{2}-16 \pi^{2} \varepsilon}\right) \\
& \leq \frac{1}{2}\left(\mathscr{H}_{\varepsilon}(v)+\sqrt{\mathscr{H}_{\varepsilon}(v)^{2}-16 \pi^{2} \varepsilon}\right)<4 \pi,
\end{aligned}
$$

since $\mathscr{H}_{\varepsilon}(v)<\pi(4+\varepsilon)$ and $\varepsilon \leq 4$. Thus there exist $\delta>0, k_{0} \in \mathbb{N}$ such that $\mathscr{W}\left(u_{k}\right) \leq 4 \pi-\delta$ for all $k \geq k_{0}$. Lemma 1 then implies that $\left(u_{k}\right)_{k \in \mathbb{N}}$ is bounded in $C^{1}([-1,1])$ and that 
$0<\varrho \leq u_{k}(x) \leq \frac{1}{\varrho}$ for a suitable $\varrho>0$. Then one sees from (7) that a bound for $\mathscr{W}\left(u_{k}\right)$ also yields an $L^{2}$-bound for $\left(u_{k}^{\prime \prime}\right)_{k \in \mathbb{N}}$. Arguing as in the proof of [12, Theorem 3.9] we obtain a subsequence, again denoted by $\left(u_{k}\right)_{k \in \mathbb{N}}$, and $u \in N_{\alpha}$ such that

$$
u_{k} \rightarrow u \text { in } H^{2}(-1,1), \quad u_{k} \rightarrow u \text { in } C^{1}([-1,1])
$$

and it is easily seen that $u$ is a minimiser of $\mathscr{H}_{\varepsilon}$. The fact that $u$ belongs to $C^{\infty}([-1,1])$ can be shown by a straightforward adaptation of the corresponding argument in the proof of [12, Theorem 3.9].

In order to apply Theorem 1 we look for suitable functions $v \in N_{\alpha}$ such that $\mathscr{H}_{\varepsilon}(v)<$ $\pi(4+\varepsilon)$. For the simple choice $v \equiv \alpha$ we find that

$$
\mathscr{H}_{\varepsilon}(v)=\frac{\pi}{\alpha}+4 \pi \alpha \varepsilon
$$

As a second useful comparison function we define (see [23, Lemma 8.6])

$$
v(x):= \begin{cases}\sqrt{r^{2}-x^{2}}, & |x|<x_{0} \\ \alpha \cosh \left(\frac{|x|-1}{\alpha}\right), & x_{0} \leq|x| \leq 1,\end{cases}
$$

where $r=\sqrt{x_{0}^{2}+\alpha^{2} \cosh \left(\frac{x_{0}-1}{\alpha}\right)^{2}}$ and $x_{0} \in\left(\frac{1}{2}, 1\right)$ is chosen in such a way that

$x_{0}+\alpha \cosh \left(\frac{x_{0}-1}{\alpha}\right) \sinh \left(\frac{x_{0}-1}{\alpha}\right)=0, \quad x+\alpha \cosh \left(\frac{x-1}{\alpha}\right) \sinh \left(\frac{x-1}{\alpha}\right)>0, x \in\left(x_{0}, 1\right]$.

This condition means that the normal to the cosh-function through the point $\left(x_{0}, v\left(x_{0}\right)\right)$ intersects the $x$-axis in the origin. One may observe that

$$
x \mapsto x+\alpha \cosh \left(\frac{x-1}{\alpha}\right) \sinh \left(\frac{x-1}{\alpha}\right)
$$

is strictly increasing on $[0,1]$, strictly negative for $x=\frac{1}{2}$ and strictly positive for $x=1$. The choice of $x_{0}$ and $r$ ensures that $v \in N_{\alpha}$ and that $r \leq \sqrt{1+\alpha^{2}}$. Since $H=0$ for $x_{0} \leq|x| \leq 1$, we obtain with the help of (7) that

$$
\begin{aligned}
\mathscr{H}_{\varepsilon}(v)= & \frac{\pi}{2} \int_{-x_{0}}^{x_{0}}\left(\frac{1}{v(x) \sqrt{1+v^{\prime}(x)^{2}}}+\frac{v^{\prime \prime}(x)}{\left(1+v^{\prime}(x)^{2}\right)^{3 / 2}}\right)^{2} v(x) \sqrt{1+v^{\prime}(x)^{2}} \mathrm{~d} x \\
& -\left.2 \pi \frac{v^{\prime}(x)}{\sqrt{1+v^{\prime}(x)^{2}}}\right|_{-x_{0}} ^{x_{0}} \\
& +4 \pi \varepsilon x_{0} r+2 \pi \alpha \varepsilon\left(1-x_{0}+\frac{\alpha}{2} \sinh \left(\frac{2\left(1-x_{0}\right)}{\alpha}\right)\right) \\
< & 4 \pi \tanh \left(\frac{1-x_{0}}{\alpha}\right)+\varepsilon\left(4 \pi \sqrt{1+\alpha^{2}}+\pi \alpha+\pi \alpha^{2} \sinh \left(\frac{1}{\alpha}\right)\right) \\
< & 4 \pi \tanh \left(\frac{1}{2 \alpha}\right)+\varepsilon\left(4 \pi \sqrt{1+\alpha^{2}}+\pi \alpha+\pi \alpha^{2} \sinh \left(\frac{1}{\alpha}\right)\right),
\end{aligned}
$$

since $\frac{1}{2}<x_{0}<1$ and

$$
\frac{1}{v(x) \sqrt{1+v^{\prime}(x)^{2}}}+\frac{v^{\prime \prime}(x)}{\left(1+v^{\prime}(x)^{2}\right)^{3 / 2}}=0, \quad x \in\left(-x_{0}, x_{0}\right) .
$$

From the above calculations we infer the following existence result: 
Theorem 2 The functional $\mathscr{H}_{\varepsilon}$ attains a minimum on $N_{\alpha}$, if one of the following conditions is satisfied:

i) $\alpha>\frac{1}{4}$ and $0 \leq \varepsilon<\frac{1}{\alpha}$;

ii) $\alpha>0$ and $0 \leq \varepsilon \leq \frac{1-\tanh \left(\frac{1}{2 \alpha}\right)}{\sqrt{1+\alpha^{2}}+\frac{\alpha}{4}+\frac{\alpha^{2}}{4} \sinh \left(\frac{1}{\alpha}\right)-\frac{1}{4}}$.

Proof The result follows from (15), (16) and Theorem 1.

\section{Perturbation of Helfrich cylinders}

Throughout this section we fix for a given $\alpha>0$

$$
\varepsilon_{\alpha}:=\frac{1}{4 \alpha^{2}}
$$

as the parameter where the Helfrich cylinder

$$
u_{\varepsilon_{\alpha}}(x) \equiv \alpha
$$

is the unique minimiser of $\mathscr{H}_{\varepsilon_{\alpha}}$ (see [43, Lemma 4.1]). Let us define

$$
F(\varepsilon, u):=\Delta_{S} H+2 H\left(H^{2}-K\right)-2 \varepsilon H .
$$

Recalling (12) and taking into account that

$$
H\left[u_{\varepsilon_{\alpha}}\right] \equiv \frac{1}{2 \alpha}, \quad \frac{\mathrm{d}}{\mathrm{d} \delta} H\left[u_{\varepsilon_{\alpha}}+\delta \varphi\right]_{\mid \delta=0}=\frac{1}{2}\left(-\frac{1}{\alpha^{2}} \varphi-\varphi^{\prime \prime}\right)
$$

we calculate

$$
\begin{aligned}
\langle & \left.\frac{\partial F}{\partial u}\left(\varepsilon_{\alpha}, u_{\varepsilon_{\alpha}}\right), \varphi\right\rangle=\frac{\mathrm{d}}{\mathrm{d} \delta} F\left(\varepsilon_{\alpha}, \alpha+\delta \varphi\right)_{\mid \delta=0} \\
= & \frac{1}{\alpha} \frac{\mathrm{d}^{2}}{\mathrm{~d} x^{2}}\left(\alpha \frac{1}{2}\left(-\frac{1}{\alpha^{2}} \varphi-\varphi^{\prime \prime}\right)\right)+\frac{1}{4} \frac{1}{\alpha^{2}}\left(-\frac{1}{\alpha^{2}} \varphi-\varphi^{\prime \prime}\right)+\frac{1}{2 \alpha} \frac{1}{\alpha}\left(\varphi^{\prime \prime}-\frac{1}{\alpha^{2}} \varphi\right) \\
& -\frac{1}{4 \alpha^{2}}\left(-\frac{1}{\alpha^{2}} \varphi-\varphi^{\prime \prime}\right) \\
= & -\frac{1}{2}\left(\varphi^{(i v)}+\frac{1}{\alpha^{4}} \varphi\right) .
\end{aligned}
$$

Theorem 3 For every $\alpha>0$ there exists $\delta>0$ such that for all $\varepsilon \in\left(\varepsilon_{\alpha}-\delta, \varepsilon_{\alpha}+\delta\right)$ the boundary value problem (12), (13) has a smooth family of solutions $u_{\varepsilon} \in N_{\alpha} \cap C^{\infty}([-1,1])$.

Proof We apply the implicit function theorem as it can be found in [17, Theorem 15.1]. Let $X=\mathbb{R}, U=(0, \infty) \subset X, Y=C^{4}([-1,1]), V=C^{4}([-1,1],(0, \infty)) \subset Y, Z=$ $C^{0}([-1,1]) \times \mathbb{R}^{4}$. Moreover consider the function $\Phi: U \times V \rightarrow Z$ defined by

$$
\Phi(\varepsilon, u):=\left(F(\varepsilon, u), u(-1)-\alpha, u(1)-\alpha, u^{\prime}(-1), u^{\prime}(1)\right) .
$$

In view of (17) we obtain for $L: Y \rightarrow Z$ with $L(\varphi):=\left\langle\frac{\partial \Phi}{\partial u}\left(\varepsilon_{\alpha}, u_{\varepsilon_{\alpha}}\right), \varphi\right\rangle$ that

$$
L(\varphi)=\left(-\frac{1}{2}\left(\varphi^{(i v)}+\frac{1}{\alpha^{4}} \varphi\right), \varphi(-1), \varphi(1), \varphi^{\prime}(-1), \varphi^{\prime}(1)\right) .
$$


We claim that $L$ is injective. To see this, consider the boundary value problem

$$
\varphi^{(i v)}+\frac{1}{\alpha^{4}} \varphi=0 \text { in }(-1,1), \quad \varphi( \pm 1)=\varphi^{\prime}( \pm 1)=0 .
$$

The roots of the characteristic equation for the differential equation in (18) are given by $\pm \frac{1+i}{\alpha \sqrt{2}}$ and $\pm \frac{1-i}{\alpha \sqrt{2}}$ so that the functions

$$
\begin{aligned}
& \varphi_{1}(x)=\cosh \left(\frac{x}{\alpha \sqrt{2}}\right) \cos \left(\frac{x}{\alpha \sqrt{2}}\right), \varphi_{2}(x)=\sinh \left(\frac{x}{\alpha \sqrt{2}}\right) \sin \left(\frac{x}{\alpha \sqrt{2}}\right), \\
& \varphi_{3}(x)=\cosh \left(\frac{x}{\alpha \sqrt{2}}\right) \sin \left(\frac{x}{\alpha \sqrt{2}}\right), \varphi_{4}(x)=\sinh \left(\frac{x}{\alpha \sqrt{2}}\right) \cos \left(\frac{x}{\alpha \sqrt{2}}\right)
\end{aligned}
$$

form a fundamental system. Hence there exist constants $b_{1}, \ldots, b_{4} \in \mathbb{R}$ such that $\varphi(x)=$ $\sum_{j=1}^{4} b_{j} \varphi_{j}(x), x \in[-1,1]$. The four boundary conditions $\varphi( \pm 1)=\varphi^{\prime}( \pm 1)=0$ translate into the linear system $M b=0$ with $b=\left(b_{1}, \ldots, b_{4}\right)$ and $m_{1 j}=\varphi_{j}(1), m_{2 j}=$ $\varphi_{j}(-1), m_{3 j}=\frac{1}{\beta} \varphi_{j}^{\prime}(1), m_{4 j}=\frac{1}{\beta} \varphi_{j}^{\prime}(-1), j=1, \ldots, 4$, where we have abbreviated $\beta=\frac{1}{\alpha \sqrt{2}}>0$. Using the above expressions for $\varphi_{j}$ we find that

$$
M=\left(\begin{array}{cccc}
\cosh (\beta) \cos (\beta) & \sinh (\beta) \sin (\beta) & \cosh (\beta) \sin (\beta) & \sinh (\beta) \cos (\beta) \\
\cosh (\beta) \cos (\beta) & \sinh (\beta) \sin (\beta) & -\cosh (\beta) \sin (\beta) & -\sinh (\beta) \cos (\beta) \\
\sinh (\beta) \cos (\beta) & \cosh (\beta) \sin (\beta)) & \sinh (\beta) \sin (\beta) & \cosh (\beta) \cos (\beta) \\
-\cosh (\beta) \sin (\beta) & +\sinh (\beta) \cos (\beta) & +\cosh (\beta) \cos (\beta) & -\sinh (\beta) \sin (\beta) \\
-\sinh (\beta) \cos (\beta) & -\cosh (\beta) \sin (\beta)) & \sinh (\beta) \sin (\beta) & \cosh (\beta) \cos (\beta) \\
+\cosh (\beta) \sin (\beta) & -\sinh (\beta) \cos (\beta) & +\cosh (\beta) \cos (\beta) & -\sinh (\beta) \sin (\beta)
\end{array}\right)
$$

and a calculation shows that

$$
\operatorname{det}(M)=-4 \sinh ^{2}(\beta) \cosh ^{2}(\beta)+4 \sin ^{2}(\beta) \cos ^{2}(\beta)<0 .
$$

Hence $b_{1}=\cdots=b_{4}=0$, which yields $\varphi \equiv 0$, so that $L$ is injective. Furthermore, since $L$ allows for an elliptic theory, i.e. $L$ is Fredholm of index 0 , we infer that $L$ is invertible with bounded inverse. Hence we can apply the implicit function theorem to obtain solutions $u_{\varepsilon} \in N_{\alpha} \cap C^{4}([-1,1])$ for $\varepsilon$ close to $\varepsilon_{0}$. By arguing again as in the proof of Theorem 1 we also have $u_{\varepsilon} \in C^{\infty}([-1,1])$.

To study the behaviour of $u_{\varepsilon}$ for $\varepsilon$ close to $\varepsilon_{\alpha}$ we introduce

$$
r c_{\alpha}:=\left.\frac{\partial u_{\varepsilon}}{\partial \varepsilon}\right|_{\varepsilon=\varepsilon_{\alpha}} .
$$

Since $\frac{\mathrm{d}}{\mathrm{d} \varepsilon} F\left(\varepsilon, u_{\varepsilon}\right)_{\mid \varepsilon=\varepsilon_{\alpha}}=0$ we find with the help of (17) that

$$
0=\frac{\partial F}{\partial \varepsilon}\left(\varepsilon_{\alpha}, u_{\varepsilon_{\alpha}}\right)+\left\langle\frac{\partial F}{\partial u}\left(\varepsilon_{\alpha}, u_{\varepsilon_{\alpha}}\right), r c_{\alpha}\right\rangle=-\frac{1}{\alpha}-\frac{1}{2}\left(r c_{\alpha}^{(i v)}+\frac{1}{\alpha^{4}} r c_{\alpha}\right)
$$

giving rise to the following boundary value problem for $r c_{\alpha}$ :

$$
\left\{\begin{array}{l}
r c_{\alpha}^{(i v)}+\frac{1}{\alpha^{4}} r c_{\alpha}=-\frac{2}{\alpha} \quad \text { in } \quad(-1,1) \\
r c_{\alpha}( \pm 1)=r c_{\alpha}^{\prime}( \pm 1)=0 .
\end{array}\right.
$$


This problem is solved by (cf. Fig. 2)

$$
r c_{\alpha}(x)=-2 \alpha^{3}+a_{\alpha} \cosh \left(\frac{x}{\alpha \sqrt{2}}\right) \cos \left(\frac{x}{\alpha \sqrt{2}}\right)+b_{\alpha} \sinh \left(\frac{x}{\alpha \sqrt{2}}\right) \sin \left(\frac{x}{\alpha \sqrt{2}}\right)
$$

with

$$
\begin{aligned}
& a_{\alpha}=\frac{2 \alpha^{3}}{d_{\alpha}}\left[\sinh \left(\frac{1}{\alpha \sqrt{2}}\right) \cos \left(\frac{1}{\alpha \sqrt{2}}\right)+\cosh \left(\frac{1}{\alpha \sqrt{2}}\right) \sin \left(\frac{1}{\alpha \sqrt{2}}\right)\right] \\
& b_{\alpha}=-\frac{2 \alpha^{3}}{d_{\alpha}}\left[\sinh \left(\frac{1}{\alpha \sqrt{2}}\right) \cos \left(\frac{1}{\alpha \sqrt{2}}\right)-\cosh \left(\frac{1}{\alpha \sqrt{2}}\right) \sin \left(\frac{1}{\alpha \sqrt{2}}\right)\right], \\
& d_{\alpha}=\cosh \left(\frac{1}{\alpha \sqrt{2}}\right) \sinh \left(\frac{1}{\alpha \sqrt{2}}\right)+\cos \left(\frac{1}{\alpha \sqrt{2}}\right) \sin \left(\frac{1}{\alpha \sqrt{2}}\right) .
\end{aligned}
$$

Note that $d_{\alpha}=\frac{1}{2} \sinh \left(\frac{\sqrt{2}}{\alpha}\right)+\frac{1}{2} \sin \left(\frac{\sqrt{2}}{\alpha}\right)>0$. We have:

Theorem 4 Let $\alpha>0$ and $r c_{\alpha}$ as in (19). Then $r c_{\alpha}(x)<0$ for all $x \in(-1,1)$.

Proof Let us abbreviate $\beta=\frac{1}{\alpha \sqrt{2}}$. For $x \in[-1,1]$ we compute with the help of Corollary 4 from Appendix C with $a=\beta$

$$
\begin{aligned}
\frac{d_{\alpha}}{2 \alpha^{3}} r c_{\alpha}(x)= & -(\cosh (\beta) \sinh (\beta)+\cos (\beta) \sin (\beta)) \\
& +(\sinh (\beta) \cos (\beta)+\cosh (\beta) \sin (\beta)) \cosh (\beta x) \cos (\beta x) \\
& -(\sinh (\beta) \cos (\beta)-\cosh (\beta) \sin (\beta)) \sinh (\beta x) \sin (\beta x) \\
\leq & -(\cosh (\beta) \sinh (\beta)+\cos (\beta) \sin (\beta)) \\
& +(\sinh (\beta) \cos (\beta)+\cosh (\beta) \sin (\beta)) \cosh (\beta) \cos (\beta) \\
& -(\sinh (\beta) \cos (\beta)-\cosh (\beta) \sin (\beta)) \sinh (\beta) \sin (\beta)=0
\end{aligned}
$$

The statement then follows since equality only holds at $x= \pm 1$.

Theorem 5 Let $a_{c} \in\left(\pi, \frac{3}{2} \pi\right)$ denote the smallest, strictly positive solution of the equation $\tanh (x)=\tan (x)($ see Lemma 6 in Appendix $B)$ and $\alpha_{\text {crit }}=\frac{1}{a_{c} \sqrt{2}} \approx 0.18008$. Then $r c_{\alpha}^{\prime}(x)>$ 0 for all $x \in(0,1)$ and all $\alpha \geq \alpha_{\text {crit. }}$

Proof Let us abbreviate again $\beta=\frac{1}{\alpha \sqrt{2}}$. It follows from Lemma 7 in Appendix C with $a=\beta$ that for every $x \in(0,1)$

$$
\begin{aligned}
r c_{\alpha}^{\prime}(x) & =\left(b_{\alpha}-a_{\alpha}\right) \cosh (\beta x) \sin (\beta x)+\left(b_{\alpha}+a_{\alpha}\right) \sinh (\beta x) \cos (\beta x) \\
& =\frac{2 \alpha^{3}}{d_{\alpha}}[\sinh (\beta x) \cos (\beta x) \cosh (\beta) \sin (\beta)-\sinh (\beta) \cos (\beta) \cosh (\beta x) \sin (\beta x)]>0
\end{aligned}
$$

since $0<\beta \leq a_{c}$.

Remark 1 The end of the proof of Lemma 7 shows that for $\alpha \in\left(0, \alpha_{\text {crit }}\right)$ and $\alpha \searrow 0$, we observe an increasing number of sign changes of $r c_{\alpha}^{\prime}$. 


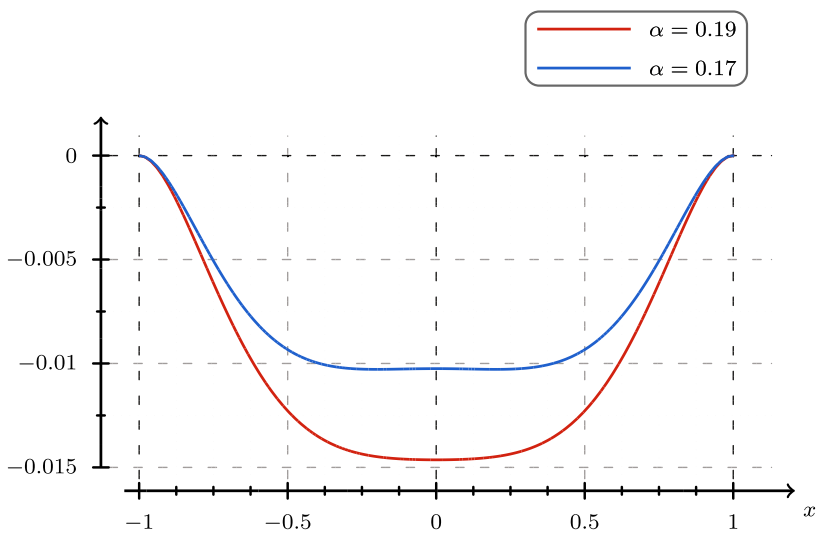

Fig. 2 Plots of the rate of change function $r c_{\alpha}$ for two different values of $\alpha$ around the value $\alpha_{\text {crit }} \approx 0.18008$ in accordance with Theorems 4 and 5. The function for $\alpha=0.17$ develops an oscillatory behaviour with oscillations starting at the origin $x=0$

\section{Existence of minimisers via gluing techniques}

We start with a discussion of symmetric positive profile curves satisfying $H \equiv 0$, i.e.

$$
\frac{1}{u(x) \sqrt{1+u^{\prime}(x)^{2}}}-\frac{u^{\prime \prime}(x)}{\left(1+u^{\prime}(x)^{2}\right)^{3 / 2}}=0 \quad \text { in }[-1,1] .
$$

The corresponding solutions are the catenaries

$$
w_{c}(x):=c \cosh \left(\frac{x}{c}\right), \quad x \in[-1,1]
$$

with boundary values $c \cosh \left(\frac{1}{c}\right)$ and surface area

$$
\begin{aligned}
\mathscr{A}\left(w_{c}\right) & =2 \pi \int_{-1}^{1} w_{c}(x) \sqrt{1+w_{c}^{\prime}(x)^{2}} \mathrm{~d} x \\
& =2 \pi c+\pi c^{2} \sinh \left(\frac{2}{c}\right)=2 \pi c\left(1+c \sinh \left(\frac{1}{c}\right) \cosh \left(\frac{1}{c}\right)\right) .
\end{aligned}
$$

A discussion of the function $c \mapsto c \cosh \left(\frac{1}{c}\right)$ shows that

$$
c \mapsto c \cosh \left(\frac{1}{c}\right) \text { is }\left\{\begin{array}{l}
\text { strictly decreasing on }\left(0, c_{0}\right] \\
\text { strictly increasing on }\left[c_{0}, \infty\right)
\end{array}\right.
$$

where $c_{0} \approx 0.8336$ is the positive solution of the equation $c_{0}=\tanh \left(\frac{1}{c_{0}}\right)$. Setting $\alpha_{0}:=$ $c_{0} \cosh \left(\frac{1}{c_{0}}\right) \approx 1.5089$ we infer that the equation

$$
c \cosh \left(\frac{1}{c}\right)=\alpha
$$

has two solutions $c_{1}(\alpha)>c_{0}>c_{2}(\alpha)$ if $\alpha>\alpha_{0}$, one solution $c_{1}(\alpha)$ if $\alpha=\alpha_{0}$ and no solution for $\alpha<\alpha_{0}$. Although presumably folklore and asymptotically obvious for $\alpha \rightarrow \infty$, 
we could not locate an easy reference for the following statement which is quite important in what follows:

$$
\mathscr{A}\left(w_{c_{1}(\alpha)}\right)<\mathscr{A}\left(w_{c_{2}(\alpha)}\right), \quad \alpha>\alpha_{0} .
$$

For the reader's convenience we give a proof in Appendix A.

In what follows we shall write

$$
c_{\alpha}:=c_{1}(\alpha), \quad v_{\alpha}:=w_{c_{\alpha}}, \hat{\varepsilon}_{\alpha}:=\frac{1}{4 c_{\alpha}^{2}} .
$$

In particular it follows from (24) that

$$
\left(\alpha_{0}, \infty\right) \ni \alpha \mapsto c_{\alpha} \quad \text { is strictly increasing. }
$$

In order to make the role of $v_{\alpha}$ in the minimisation of $\mathscr{A}$ more precise it is convenient to relax the class of profile curves and consider for a given boundary value $\alpha>0$

$\mathscr{C}_{\alpha}:=\left\{\gamma \in C^{0,1}\left([-1,1], \mathbb{R}^{2}\right): \gamma_{2}(t) \geq 0, \gamma( \pm 1)=( \pm 1, \alpha), \dot{\gamma}(t) \neq 0\right.$ a.e. in $\left.[-1,1]\right\}$,

where the area functional is now given by

$$
\mathscr{A}(\gamma):=2 \pi \int_{-1}^{1} \gamma_{2}(t)|\dot{\gamma}(t)| \mathrm{d} t .
$$

In order to formulate the main result concerning the minimisation of $\mathscr{A}$ over $\mathscr{C}_{\alpha}$ we introduce for $\alpha>0$ the Goldschmidt solution $\gamma_{\alpha}$ as a $C^{0,1}$ parametrisation of the polygon $P_{-} Q_{-} Q_{+} P_{+}$, where $P_{ \pm}=( \pm 1, \alpha), Q_{ \pm}=( \pm 1,0)$. As a geometric object the Goldschmidt solution corresponds to two disks with radius $\alpha$ and centers $Q_{-}, Q_{+}$and its surface area is given by $\mathscr{A}\left(\gamma_{\alpha}\right)=2 \pi \alpha^{2}$. Appendix A shows also that $\mathscr{A}\left(v_{\alpha}\right)=\mathscr{A}\left(\gamma_{\alpha}\right)$ if and only if $\alpha=\alpha_{m}=$ $c_{m} \cosh \left(\frac{1}{c_{m}}\right) \approx 1.895$, where $c_{m} \approx 1.564$ is the unique solution of the equation

$$
\frac{2}{c}=1+e^{-2 / c} \text {. }
$$

For $\alpha>\alpha_{m}$ we have $\mathscr{A}\left(v_{\alpha}\right)<\mathscr{A}\left(\gamma_{\alpha}\right)$ while $\mathscr{A}\left(\gamma_{\alpha}\right)<\mathscr{A}\left(v_{\alpha}\right)$ for $\alpha<\alpha_{m}$. The following result is then a special case of Theorem 1 in [25, Chapter 8, Section 4.3]:

Theorem 6 (Absolute minimisers of the area functional) For every $\alpha>0$ the variational problem

$$
\min _{\gamma \in \mathscr{C}_{\alpha}} \mathscr{A}(\gamma)=2 \pi \int_{-1}^{1} \gamma_{2}(t)|\dot{\gamma}(t)| \mathrm{d} t
$$

has a solution. This solution is furnished by the catenary $t \mapsto\left(t, v_{\alpha}(t)\right)$ if $\alpha>\alpha_{m}$, the Goldschmidt solution $\gamma_{\alpha}$ if $\alpha<\alpha_{m}$ and both of them if $\alpha=\alpha_{m}$. Except for reparametrisation there are no further solutions.

We use the above result in order to prove the following lemma.

Lemma 2 Suppose that $\alpha \geq \alpha_{m}$. For $u \in N_{\alpha}$ there exists $v \in N_{\alpha}$ such that $v^{\prime}(x)<v_{\alpha}^{\prime}(x)$ for all $x \in(0,1]$ and $\mathscr{H}_{\varepsilon}(v) \leq \mathscr{H}_{\varepsilon}(u)$. If $u$ has finitely many critical points, then the same holds for $v$. In particular we have that $v(x)>v_{\alpha}(x)$ for all $x \in(-1,1)$. 


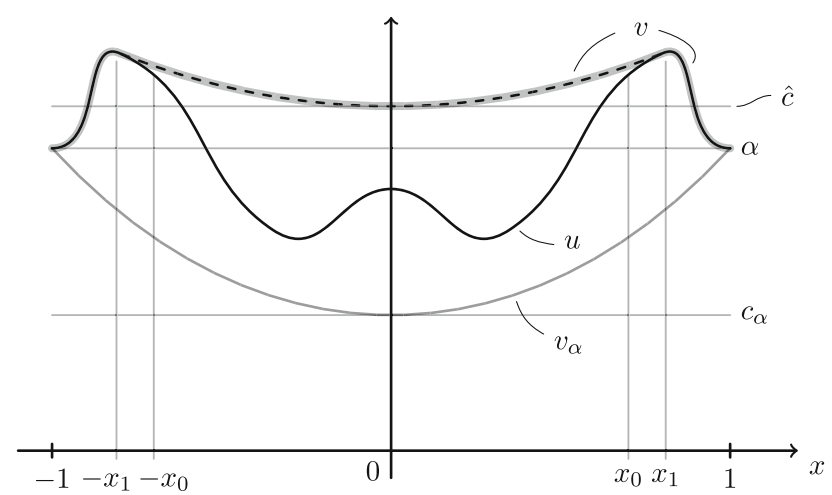

Fig. 3 Construction of $v$ by attaching an appropriate catenary to $u$ at $-x_{1}$ and $x_{1}$

Proof Since $u^{\prime}(1)=0<v_{\alpha}^{\prime}(1)$, there exists $x_{0} \in[0,1)$ such that

$$
\forall x \in\left(x_{0}, 1\right]: \quad u^{\prime}(x)<v_{\alpha}^{\prime}(x) \quad \text { and } \quad u^{\prime}\left(x_{0}\right)=v_{\alpha}^{\prime}\left(x_{0}\right) .
$$

If $x_{0}=0$ set $v=u$, otherwise define

$$
\hat{c}:=\inf \left\{c \geq c_{\alpha} \mid w_{c}(x)>u(x) \text { for all } x \in\left[x_{0}, 1\right]\right\} .
$$

Clearly,

$$
\forall x \in\left[x_{0}, 1\right]: \quad w_{\hat{c}}(x) \geq u(x), \quad \text { and } \quad \exists x_{1} \in\left[x_{0}, 1\right]: \quad w_{\hat{c}}\left(x_{1}\right)=u\left(x_{1}\right) .
$$

Suppose that $\hat{c}=c_{\alpha}$. Then, in view of (26) and (29) we have that $v_{\alpha}(x) \geq u(x)$ for $x \in\left[x_{0}, 1\right]$ and hence $v_{\alpha}^{\prime}(1) \leq u^{\prime}(1)=0$, a contradiction, so that $\hat{c}>c_{\alpha}$. Assume next that $x_{1} \in\left\{x_{0}, 1\right\}$. Using again (26), (29) as well as (28) we infer that

$$
\begin{gathered}
\sinh \left(\frac{x_{0}}{c_{\alpha}}\right)=v_{\alpha}^{\prime}\left(x_{0}\right)=u^{\prime}\left(x_{0}\right) \leq w_{\hat{c}}^{\prime}\left(x_{0}\right)=\sinh \left(\frac{x_{0}}{\hat{c}}\right), \text { if } x_{1}=x_{0} ; \\
c_{\alpha} \cosh \left(\frac{1}{c_{\alpha}}\right)=\alpha=u(1)=w_{\hat{c}}(1)=\hat{c} \cosh \left(\frac{1}{\hat{c}}\right), \text { if } x_{1}=1 ;
\end{gathered}
$$

which is in either case a contradiction to $\hat{c}>c_{\alpha}$. As a result we deduce that $x_{1} \in\left(x_{0}, 1\right)$, which implies that $w_{\hat{c}}^{\prime}\left(x_{1}\right)=u^{\prime}\left(x_{1}\right)$. The function

$$
v(x):=\left\{\begin{array}{l}
u(x), \quad x \in\left[-1,-x_{1}\right] \cup\left[x_{1}, 1\right] \\
w_{\hat{c}}(x), x \in\left(-x_{1}, x_{1}\right)
\end{array}\right.
$$

(see Fig. 3) then belongs to $N_{\alpha}$. Since the catenoid given by $w_{\hat{c}}$ has zero mean curvature we have

$$
\begin{aligned}
& \mathscr{H}_{\varepsilon}(v)-\mathscr{H}_{\varepsilon}(u) \leq 2 \pi \varepsilon \int_{-x_{1}}^{x_{1}} w_{\hat{c}}(x) \sqrt{1+w_{\hat{c}}^{\prime}(x)^{2}} \mathrm{~d} x-2 \pi \varepsilon \int_{-x_{1}}^{x_{1}} u(x) \sqrt{1+u^{\prime}(x)^{2}} \mathrm{~d} x \\
& =2 \pi \varepsilon x_{1} \int_{-1}^{1} w_{\hat{c}}\left(x_{1} x\right) \sqrt{1+w_{\hat{c}}^{\prime}\left(x_{1} x\right)^{2}} \mathrm{~d} x-2 \pi \varepsilon x_{1} \int_{-1}^{1} u\left(x_{1} x\right) \sqrt{1+u^{\prime}\left(x_{1} x\right)^{2}} \mathrm{~d} x \\
& =2 \pi \varepsilon x_{1}^{2}\left\{\int_{-1}^{1} w_{\hat{c} / x_{1}}(x) \sqrt{1+w_{\hat{c} / x_{1}}^{\prime}(x)^{2}} \mathrm{~d} x-\int_{-1}^{1} \hat{u}(x) \sqrt{1+\hat{u}^{\prime}(x)^{2}} \mathrm{~d} x\right\},
\end{aligned}
$$




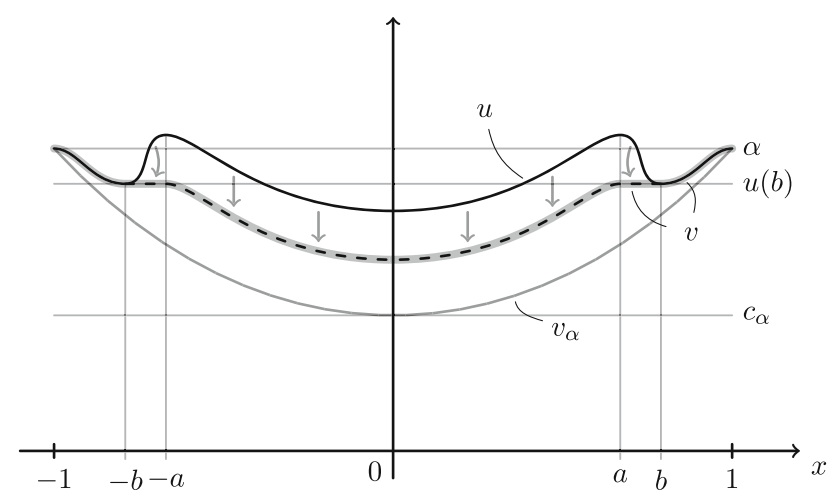

Fig. 4 Construction of the function $v$ from $u$ by inserting a cylinder in the intervals $[-b,-a],[a, b]$ and translating the inner part to match the cylinder

where $\hat{u}(x)=\frac{1}{x_{1}} u\left(x_{1} x\right), x \in[-1,1]$. Since $\frac{\hat{c}}{x_{1}}>\hat{c}>c_{\alpha} \geq c_{0}$, it follows from (27) that

$$
\frac{\hat{c}}{x_{1}} \cosh \left(\frac{x_{1}}{\hat{c}}\right)>c_{\alpha} \cosh \left(\frac{1}{c_{\alpha}}\right)=\alpha \geq \alpha_{m}
$$

and Theorem 6 implies that $w_{\hat{c} / x_{1}}$ is a global minimum for the area functional in the class $\mathscr{C}_{\hat{\alpha}}$ with $\hat{\alpha}=\frac{\hat{c}}{x_{1}} \cosh \left(\frac{x_{1}}{\hat{c}}\right)$. Taking into account that $w_{\hat{c} / x_{1}}( \pm 1) \stackrel{\text { def }}{=} \frac{\hat{c}}{x_{1}} \cosh \left(\frac{x_{1}}{\hat{c}}\right)=\frac{1}{x_{1}} w_{\hat{c}}\left(x_{1}\right) \stackrel{(29)}{=}$ $\frac{1}{x_{1}} u\left(x_{1}\right) \stackrel{\text { def }}{=} \hat{u}( \pm 1)$ we hence infer that

$$
\int_{-1}^{1} w_{\hat{c} / x_{1}}(x) \sqrt{1+w_{\hat{c} / x_{1}}^{\prime}(x)^{2}} \mathrm{~d} x \leq \int_{-1}^{1} \hat{u}(x) \sqrt{1+\hat{u}^{\prime}(x)^{2}} \mathrm{~d} x
$$

giving $\mathscr{H}_{\varepsilon}(v) \leq \mathscr{H}_{\varepsilon}(u)$. We see from the construction that $v$ has finitely many critical points if this was the case for $u$. Finally, the inequality $v(x)>v_{\alpha}(x), x \in(-1,1)$ easily follows by integration.

Lemma 3 Let $\alpha \geq \alpha_{m}$ and $\varepsilon \geq \hat{\varepsilon}_{\alpha}$. Suppose that $u \in N_{\alpha}$ has finitely many critical points and satisfies $u^{\prime}(x)<v_{\alpha}^{\prime}(x)$ for all $x \in(0,1]$. Then there exists $v \in N_{\alpha}$ such that $0 \leq v^{\prime}(x)<$ $v_{\alpha}^{\prime}(x)$ for $x \in(0,1]$ and $\mathscr{H}_{\varepsilon}(v) \leq \mathscr{H}_{\varepsilon}(u)$. In particular, we have that $v_{\alpha}(x)<v(x) \leq \alpha$ for all $x \in(-1,1)$.

Proof Let us assume that $u^{\prime}\left(x_{0}\right)<0$ for some $x_{0} \in(0,1)$. Then there exists an interval $[a, b] \subset[0,1]$ such that

$$
\forall x \in[b, 1]: \quad u^{\prime}(x) \geq 0 ; \quad \forall x \in(a, b): \quad u^{\prime}(x)<0 ; \quad u^{\prime}(a)=u^{\prime}(b)=0 .
$$

Let us define

$$
v(x):= \begin{cases}u(x), & x \in[-1,-b] \cup[b, 1] \\ u(b), & x \in(-b,-a) \cup(a, b) ; \\ u(x)-(u(a)-u(b)), & x \in[-a, a]\end{cases}
$$

see Fig. 4. Clearly, $v \in H^{2}(-1,1)$ is even with

$$
v^{\prime}(x)= \begin{cases}u^{\prime}(x), & x \in[-1,-b] \cup[b, 1] \\ 0, & x \in(-b,-a) \cup(a, b) \\ u^{\prime}(x), & x \in[-a, a]\end{cases}
$$


so that $v^{\prime}(x)<v_{\alpha}^{\prime}(x)$ for $x \in(0,1]$ and $v^{\prime}(x) \geq 0$ for $x \in[a, 1]$. As a consequence,

$$
v(x)=v(1)-\int_{x}^{1} v^{\prime}(t) \mathrm{d} t \geq \alpha-\int_{x}^{1} v_{\alpha}^{\prime}(t) \mathrm{d} t=v_{\alpha}(x) \geq c_{\alpha}, \quad x \in[0,1]
$$

and in particular $v$ is positive on $[-1,1]$, so that $v \in N_{\alpha}$. Next, since $u^{\prime}(x)<0$ for $x \in(a, b)$ we have that $u(x) \geq u(b)$ for $x \in[a, b]$ and therefore recalling (26)

$$
\frac{1}{\sqrt{4 \varepsilon}} \leq \frac{1}{\sqrt{4 \hat{\varepsilon}_{\alpha}}}=c_{\alpha} \leq v(x) \sqrt{1+v^{\prime}(x)^{2}} \leq u(x) \sqrt{1+u^{\prime}(x)^{2}}, \quad x \in[-1,1] .
$$

Combining the above inequality with the fact that $z \mapsto 4 \varepsilon z+\frac{1}{z}$ is increasing for $z \geq \frac{1}{\sqrt{4 \varepsilon}}$ we infer that

$$
\begin{aligned}
& 4 \varepsilon v(x) \sqrt{1+v^{\prime}(x)^{2}}+\frac{1}{v(x) \sqrt{1+v^{\prime}(x)^{2}}} \\
& \leq 4 \varepsilon u(x) \sqrt{1+u^{\prime}(x)^{2}}+\frac{1}{u(x) \sqrt{1+u^{\prime}(x)^{2}}}, \quad x \in[-1,1] .
\end{aligned}
$$

Furthermore, in view of the definition of $v$ and the fact that $v(x) \leq u(x)$ for $x \in[-b, b]$ we have

$$
\frac{v(x) v^{\prime \prime}(x)^{2}}{\left(1+v^{\prime}(x)^{2}\right)^{5 / 2}} \leq \frac{u(x) u^{\prime \prime}(x)^{2}}{\left(1+u^{\prime}(x)^{2}\right)^{5 / 2}}, \quad \text { for all } x \in[-1,1] \backslash\{ \pm a, \pm b\} .
$$

If we insert (33) and (34) into (8) we deduce that $\mathscr{H}_{\varepsilon}(v) \leq \mathscr{H}_{\varepsilon}(u)$. Note that $v_{\mid[-a, a]}$ has less critical points than $u$. If $v^{\prime}\left(x_{1}\right)<0$ for some $x_{1} \in(0, a)$ we can repeat the above procedure on $[-a, a]$ until we obtain a function with the desired properties in finitely many steps.

Remark 2 Lemma 3 is an improvement of [43, Lemma 4.8] thanks to the better bound (32) from below. There the condition on $\alpha$ and $\varepsilon$ is given by

$$
\frac{4 \varepsilon \alpha \sigma(\alpha-\sigma)}{4 \varepsilon \alpha^{2}+1} \geq 1
$$

where $\sigma \in(0, \alpha)$ is some appropriate constant. But equation (35) implies $4 \leq 4 \frac{4 \varepsilon \alpha\left(\alpha^{2} / 4\right)}{4 \varepsilon \alpha^{2}}=$ $\alpha$. With the better bounds in Theorem 7 we are able to decrease this range down to $\alpha_{m}$ (and to admit arbitrarily large $\varepsilon$ ).

Theorem 7 Assume that $\alpha \geq \alpha_{m}$ and that $\varepsilon \geq \hat{\varepsilon}_{\alpha}$. Then the Helfrich functional $\mathscr{H}_{\varepsilon}$ admits a minimiser $u \in N_{\alpha}$, which satisfies

$$
\begin{aligned}
& \forall x \in(0,1): \quad 0<u^{\prime}(x)<v_{\alpha}^{\prime}(x) ; \\
& \forall x \in(-1,1): \quad v_{\alpha}(x)<u(x)<\alpha .
\end{aligned}
$$

The function $u$ belongs to $C^{\infty}([-1,1])$ and solves the Dirichlet problem (12), (13). Furthermore, there exists a constant $c \in \mathbb{R}$ such that

$$
\frac{u(x) u^{\prime}(x) H^{\prime}(x)}{1+u^{\prime}(x)^{2}}+\frac{u(x) H(x)^{2}}{\sqrt{1+u^{\prime}(x)^{2}}}-\frac{H(x)}{1+u^{\prime}(x)^{2}}-\varepsilon \frac{u(x)}{\sqrt{1+u^{\prime}(x)^{2}}}=c, \quad x \in[-1,1] .
$$

Proof Let $\left(\tilde{u}_{k}\right)_{k \in \mathbb{N}} \subset N_{\alpha}$ be a minimising sequence, which we may assume to consist of functions with only finitely many critical points (e.g. polynomials). In view of Lemma 2 and Lemma 3 we may pass to a minimising sequence $\left(u_{k}\right)_{k \in \mathbb{N}} \subset N_{\alpha}$ such that

$$
\forall x \in(0,1]: \quad 0 \leq u_{k}^{\prime}(x) \leq v_{\alpha}^{\prime}(x) \quad \text { and } \quad \forall x \in[-1,1]: \quad v_{\alpha}(x) \leq u_{k}(x) \leq \alpha,
$$


so that $\left(u_{k}\right)_{k \in \mathbb{N}}$ is bounded in $C^{1}([-1,1])$. Arguing as in the proof of Theorem 1 we obtain the existence of a minimiser $u \in N_{\alpha}$ of $\mathscr{H}_{\varepsilon}$, which is even in $C^{\infty}([-1,1])$ thanks to elliptic regularity for the Euler-Lagrange equation. Furthermore, $u^{\prime}(x) \geq 0$ for $x \in[0,1]$. Since $u$ is a critical point of the Helfrich functional, it satisfies (12), (13) as shown in Sect. 2. Let us next denote the left hand side of (38) by $M[u]$. Using Lemma 4 in Appendix B together with (12) we obtain that $\frac{\mathrm{d}}{\mathrm{d} x} M[u](x)=0$ which implies (38).

Let us next prove the strict inequality $u^{\prime}(x)>0, x \in(0,1)$. If $\hat{x} \in[0,1]$ is a point satisfying $u^{\prime}(\hat{x})=0$, then $(38)$ yields

$$
\frac{u(\hat{x})}{4}\left(\frac{1}{u(\hat{x})}-u^{\prime \prime}(\hat{x})\right)^{2}-\frac{1}{2}\left(\frac{1}{u(\hat{x})}-u^{\prime \prime}(\hat{x})\right)-\varepsilon u(\hat{x})=c,
$$

which simplifies to

$$
\frac{1}{4 u(\hat{x})}+\varepsilon u(\hat{x})=-c+\frac{u(\hat{x}) u^{\prime \prime}(\hat{x})^{2}}{4} .
$$

Let us assume that there exists $x_{0} \in(0,1)$ with $u^{\prime}\left(x_{0}\right)=0$. Since we already know that $u^{\prime} \geq 0$ in $[0,1]$ we infer that $u^{\prime \prime}\left(x_{0}\right)=0$. Using (39) for $\hat{x}=0$ and $\hat{x}=x_{0}$ we obtain

$$
\frac{1}{4 u(0)}+\varepsilon u(0)=-c+\frac{u(0) u^{\prime \prime}(0)^{2}}{4} \geq-c=\frac{1}{4 u\left(x_{0}\right)}+\varepsilon u\left(x_{0}\right) \geq \frac{1}{4 u(0)}+\varepsilon u(0),
$$

where the last inequality follows since $z \mapsto \frac{1}{4 z}+\varepsilon z$ is strictly increasing for $z \geq \frac{1}{\sqrt{4 \varepsilon}}$ and $u\left(x_{0}\right) \geq u(0) \geq c_{\alpha} \geq \frac{1}{\sqrt{4 \varepsilon}}$. In particular, $u(0)=u\left(x_{0}\right)$ and therefore $u$ is constant on $\left[0, x_{0}\right]$. Standard ODE theory applied to (12) then implies that $u$ is constant on $[0,1]$ and hence $u \equiv \alpha$ in view of (13). This implies that $H \equiv \frac{1}{2 \alpha}$ and then again by (12) that $\varepsilon=\frac{1}{4 \alpha^{2}}$. Using the relation $\alpha=c_{\alpha} \cosh \left(\frac{1}{c_{\alpha}}\right)$ we then obtain

$$
\varepsilon=\frac{1}{4 \alpha^{2}}<\frac{1}{4 c_{\alpha}^{2}}=\hat{\varepsilon}_{\alpha}
$$

contradicting our assumption that $\varepsilon \geq \hat{\varepsilon}_{\alpha}$. Thus, $u^{\prime}(x)>0$ for $x \in(0,1)$. Since $u$ has only finitely many critical points we may assume in view of Lemma 2 and Lemma 3 that $u$ is a minimiser with the additional property that $0<u^{\prime}(x)<v_{\alpha}^{\prime}(x)$ for $x \in(0,1)$, which is (36). The inequalities in (37) then follow immediately.

Corollary 2 Assume that $\alpha \geq \alpha_{m}$. Then for any $\varepsilon \geq 0$ the Helfrich functional $\mathscr{H}_{\varepsilon}$ admits a minimiser $u \in N_{\alpha}$, which belongs to $C^{\infty}([-1,1])$ and solves the Dirichlet problem (12), (13).

Proof Since $4 c>\cosh \left(\frac{1}{c}\right)$ for $c \geq c_{m}$, we have $\frac{1}{\alpha}>\hat{\varepsilon}_{\alpha}$ for all $\alpha \geq \alpha_{m}$. Combining Theorems 1 and 7 yields the claim.

Let us next investigate the behaviour of minimisers as $\varepsilon \rightarrow \infty$.

Corollary 3 Let $\alpha \geq \alpha_{m}$ and $\left(u_{\varepsilon}\right)_{\varepsilon \geq \hat{\varepsilon}_{\alpha}}$ a sequence of minimisers of $\mathscr{H}_{\varepsilon}$ as obtained in Theorem 7. Then, $u_{\varepsilon} \rightarrow v_{\alpha}$ in $W^{1, p}(-1,1)$ as $\varepsilon \rightarrow \infty$ for all $1 \leq p<\infty$.

Proof Let $\left(\varepsilon_{k}\right)_{k \in \mathbb{N}}$ be an arbitrary sequence with $\varepsilon_{k} \geq \hat{\varepsilon}_{\alpha}$ and $\varepsilon_{k} \rightarrow \infty, k \rightarrow \infty$. Abbreviating $u_{k}=u_{\varepsilon_{k}}$ we infer from (36), (37) that $\left(u_{k}\right)_{k \in \mathbb{N}}$ is bounded in $C^{1}([-1,1])$. Thus, there exists a subsequence, again denoted by $\left(u_{k}\right)_{k \in \mathbb{N}}$, and $u \in W^{1, \infty}(-1,1)$ such that

$$
u_{k} \rightarrow u \text { in } H^{1}(-1,1) \text { and } u_{k} \rightarrow u \text { in } C^{0}([-1,1]) \text {. }
$$


In order to identify $u$ we claim that $u$ is a minimiser of $\mathscr{A}$ in the class

$$
C_{\alpha}=\left\{v \in C^{0,1}([-1,1]) \mid v \text { is even, } v>0 \text { in }[-1,1], v( \pm 1)=\alpha\right\} .
$$

To see this, let $v \in C_{\alpha}$ and fix $0<\delta \leq \frac{1}{2} \min _{[-1,1]} v$. Since $v-\alpha \in H_{0}^{1}(-1,1)$ there exists $\zeta_{\delta} \in C_{0}^{\infty}(-1,1)$ (i.e. smooth and compactly supported in $(-1,1)$ ), which is even and satisfies $\left\|v-\alpha-\zeta_{\delta}\right\|_{H^{1}} \leq \delta$. As a result

$$
\begin{aligned}
\alpha+\zeta_{\delta}(x) & \geq v(x)-\max _{[-1,1]}\left|v-\alpha-\zeta_{\delta}\right| \\
& \geq \min _{[-1,1]} v-\| v-\alpha \\
-\zeta_{\delta} \|_{H^{1}} & \geq \frac{1}{2} \min _{[-1,1]} v>0, \quad x \in[-1,1],
\end{aligned}
$$

so that $\alpha+\zeta_{\delta} \in N_{\alpha}$. Thus, $\mathscr{H}_{\varepsilon_{k}}\left(u_{k}\right) \leq \mathscr{H}_{\varepsilon_{k}}\left(\alpha+\zeta_{\delta}\right)$, which implies that

$$
\mathscr{A}\left(u_{k}\right) \leq \mathscr{A}\left(\alpha+\zeta_{\delta}\right)+\frac{1}{\varepsilon_{k}} \mathscr{W}\left(\alpha+\zeta_{\delta}\right), k \in \mathbb{N} .
$$

Sending $k \rightarrow \infty$ we infer with the help of the sequential weak lower semicontinuity of $\mathscr{A}$ in $H^{1}(-1,1)$ that

$$
\mathscr{A}(u) \leq \mathscr{A}\left(\alpha+\zeta_{\delta}\right) \leq \mathscr{A}(v)+C \delta .
$$

Since $\delta$ can be chosen arbitrarily small we deduce that $\mathscr{A}(u)=\min _{v \in C_{\alpha}} \mathscr{A}(v)$ and hence

$$
\int_{-1}^{1} \varphi(x) \sqrt{1+u^{\prime}(x)^{2}} \mathrm{~d} x+\int_{-1}^{1} \frac{u(x) u^{\prime}(x) \varphi^{\prime}(x)}{\sqrt{1+u^{\prime}(x)^{2}}} \mathrm{~d} x=0 \quad \text { for all } \varphi \in C_{0}^{\infty}(-1,1) .
$$

Note that the above relation is first obtained for all even $\varphi \in C_{0}^{\infty}(-1,1)$ and then extended to arbitrary $\varphi \in C_{0}^{\infty}(-1,1)$ using a splitting into an even and an odd part. Hence $u$ is a weak solution of (22) and it is not difficult to see that $u \in C^{2}([-1,1])$, so that $u \equiv w_{c_{1}(\alpha)}$ or $u \equiv w_{c_{2}(\alpha)}$. Recalling that $\mathscr{A}\left(w_{c_{1}(\alpha)}\right)<\mathscr{A}\left(w_{c_{2}(\alpha)}\right)$ we deduce that $u=w_{c_{1}(\alpha)}=v_{\alpha}$ and hence $\left(u_{k}\right)_{k \in \mathbb{N}}$ converges uniformly to $v_{\alpha}$. Furthermore, recalling that $0 \leq u_{k}^{\prime}(x) \leq$ $v_{\alpha}^{\prime}(x), x \in[0,1]$ we have that

$$
\left\|u_{k}^{\prime}-v_{\alpha}^{\prime}\right\|_{L^{1}(-1,1)}=2 \int_{0}^{1}\left(v_{\alpha}^{\prime}(x)-u_{k}^{\prime}(x)\right) \mathrm{d} x=2\left(u_{k}(0)-v_{\alpha}(0)\right) \rightarrow 0, k \rightarrow \infty .
$$

This implies that $u_{k}^{\prime} \rightarrow v_{\alpha}^{\prime}$ in $L^{p}(-1,1)$ for all $1 \leq p<\infty$ since $\left(u_{k}^{\prime}\right)_{k \in \mathbb{N}}$ is uniformly bounded. A standard argument then shows that the whole sequence converges to $v_{\alpha}$ in $W^{1, p}(-1,1)$ for every $1 \leq p<\infty$.

\section{Summary and outlook}

In this article we studied the Dirichlet problem $(12,13)$ for Helfrich surfaces of revolution depending on the parameters $\alpha>0$ for the boundary value and the weight factor $\varepsilon \geq 0$. Fig. 1 in the introduction gives an overview over the existence results for solutions and in particular minimisers of the Helfrich functional.

According to Corollary 2 we have the existence of minimisers of $\mathscr{H}_{\varepsilon}$ in $N_{\alpha} \cap C^{\infty}([-1,1])$ for all $\alpha \geq \alpha_{m}$ and for all $\varepsilon \geq 0$. On the other hand, with Theorem 2(ii) we can ensure that for every $\alpha>0$ and $\varepsilon \geq 0$ less than a sufficiently small $\varepsilon_{0}(\alpha)$ there exists a Helfrich 
minimiser. Our hope was to be able to prove existence of minimisers also for $\alpha<\alpha_{m}$ and for all $\varepsilon \geq 0$. This turned out to be a hard task (too hard for us) because minimisers tend to develop oscillations being created around $x=0$ and therefore gluing techniques do not seem to work. A first attempt to study this domain of parameters was done in Sect. 4. In particular Theorem 5, the analysis in Appendix C and Fig. 2 show that for $\alpha<\alpha_{\text {crit }} \approx 0.18008$ oscillatory behaviour of the rate of change function occurs, leading us to anticipate a similar behaviour for minimisers in the vicinity of the Helfrich cylinders. We conjecture that for any $\alpha<\alpha_{m}$ there will be some $\tilde{\varepsilon}_{\alpha}$ such that for $\varepsilon>\tilde{\varepsilon}_{\alpha}$ (except possibly $\varepsilon_{\alpha}$ ) all minimisers show oscillatory behaviour around the cylinder $x \mapsto \frac{1}{2 \sqrt{\varepsilon}}$. Theorem 5 and Lemma 7 indicate that for $\alpha \in\left(0, \alpha_{\text {crit }}\right)$ one may expect that even $\tilde{\varepsilon}_{\alpha}<\varepsilon_{\alpha}$. This expectation is supported by numerical experiments.

The benefit of using gluing techniques like those we used in Sect. 5 is that we obtain qualitative results for the minimisers. In Theorem 7 we found that for $\alpha>\alpha_{m}$ and $\varepsilon>\hat{\varepsilon}_{\alpha}$ the minimisers lie below the Helfrich cylinder, i.e. its boundary value at $\alpha$. We expect this to be true for all $\alpha>0$ and $\varepsilon>\varepsilon_{\alpha}$. On the other hand we do not know anything for the regime $0<\varepsilon<\varepsilon_{\alpha}$ other than the linearisation results from Sect. 4 which indicate that minimisers start to grow as $\varepsilon$ decreases. Thus we expect for these minimisers to lie above the Helfrich cylinder and possibly below the Willmore minimisers.

To conclude, let us collect some open problems:

1. $\alpha \geq \alpha_{m}$. In Corollary 3 , do we have smooth convergence away from the boundary as $\varepsilon \nearrow \infty$, i.e. in any $C_{\mathrm{loc}}^{m}(-1,1)$ ?

2. $\alpha<\alpha_{m}$ : Existence of minimisers for all $\varepsilon>0$ ?

3. $\alpha<\alpha_{m}$ : Analogously to Corollary 3 , does the sequence of minimisers $\left(u_{\varepsilon}\right)_{\varepsilon>0}$ converge to the Goldschmidt solution as $\varepsilon \nearrow \infty$ ? In which sense?

4. $\alpha<\alpha_{m}$ : What happens in the boundary layer as $\varepsilon \nearrow \infty$ ?

5. $0<\varepsilon<\varepsilon_{\alpha}$ : Do minimisers lie below the Willmore minimisers and above the Helfrich cylinder, i. e. $x \mapsto \alpha$ ?

6. $\varepsilon>\varepsilon_{\alpha}$ : Do minimisers lie below the Helfrich cylinder, i.e. $x \mapsto \alpha$ ?

7. Are there parameter intervals for $\alpha$ such that the values $u_{\varepsilon}(x)$ of (suitable) Helfrich minimisers for $x \in[-1,1]$ are a decreasing function in $\varepsilon$ ?

8. Can one quantify the domain where oscillatory behaviour occurs?

9. Can one expect uniqueness of minimisers, at least in some parameter regime? To our knowledge this question is completely open.

Acknowledgements We are grateful to the referee for his or her very careful reading of the manuscript and for his or her very helpful suggestions.

Funding Open Access funding enabled and organized by Projekt DEAL

Open Access This article is licensed under a Creative Commons Attribution 4.0 International License, which permits use, sharing, adaptation, distribution and reproduction in any medium or format, as long as you give appropriate credit to the original author(s) and the source, provide a link to the Creative Commons licence, and indicate if changes were made. The images or other third party material in this article are included in the article's Creative Commons licence, unless indicated otherwise in a credit line to the material. If material is not included in the article's Creative Commons licence and your intended use is not permitted by statutory regulation or exceeds the permitted use, you will need to obtain permission directly from the copyright holder. To view a copy of this licence, visit http://creativecommons.org/licenses/by/4.0/. 


\section{Appendix A: The "small" and the "large" catenoid}

For the reader's convenience we collect some arguments, which do not only show inequality (25) but also the remarks before Theorem 6 . We do not claim originality, but we could not locate a reference for what follows.

We consider $c \in(0, \infty)$ as independent variable and use as before $w_{c}$ for the corresponding catenoids and the radii of their boundary circles

$$
(0, \infty) \ni c \mapsto \alpha(c):=c \cosh \left(\frac{1}{c}\right)
$$

as a function of $c$. We calculate the first three derivatives:

$$
\begin{aligned}
\alpha^{\prime}(c) & =\cosh \left(\frac{1}{c}\right)-\frac{1}{c} \sinh \left(\frac{1}{c}\right), \\
\alpha^{\prime \prime}(c) & =\frac{1}{c^{3}} \cosh \left(\frac{1}{c}\right)>0, \\
\alpha^{\prime \prime \prime}(c) & =-\frac{3}{c^{4}} \cosh \left(\frac{1}{c}\right)-\frac{1}{c^{5}} \sinh \left(\frac{1}{c}\right)<0 .
\end{aligned}
$$

As remarked above, $\alpha^{\prime}(c)=0 \Leftrightarrow c=\tanh \left(\frac{1}{c}\right) \Leftrightarrow c=c_{0}$. The strict monotonicity of $\alpha^{\prime \prime}$ yields

$$
\forall c \in\left(0, c_{0}\right): \quad \alpha^{\prime \prime}\left(c_{0}-c\right)>\alpha^{\prime \prime}\left(c_{0}+c\right) .
$$

Using $\alpha^{\prime}\left(c_{0}\right)=0$, integration yields $\forall c \in\left(0, c_{0}\right): \quad-\alpha^{\prime}\left(c_{0}-c\right)>\alpha^{\prime}\left(c_{0}+c\right)$. After a further integration we come up with

$$
\forall c \in\left(0, c_{0}\right): \quad \alpha\left(c_{0}-c\right)>\alpha\left(c_{0}+c\right) .
$$

The idea in proving (25) consists in comparing the area of the catenoids $w_{c}$ and of the Goldschmidt solutions $\gamma_{\alpha(c)}$ :

$$
\begin{aligned}
\frac{1}{2 \pi}\left(\mathscr{A}\left(w_{c}\right)-\mathscr{A}\left(\gamma_{\alpha(c)}\right)\right) & =c+\frac{c^{2}}{2} \sinh \left(\frac{2}{c}\right)-c^{2} \cosh ^{2}\left(\frac{1}{c}\right) \\
& =c-\frac{c^{2}}{2}-\frac{c^{2}}{2} e^{-2 / c} .
\end{aligned}
$$

We hence define the function

$$
(0, \infty) \ni c \mapsto g(c):=c-\frac{c^{2}}{2}-\frac{c^{2}}{2} e^{-2 / c} .
$$

One may note that $g$ may be smoothly extended to $[0, \infty)$. As above we calculate the first three derivatives:

$$
\begin{aligned}
g^{\prime}(c) & =1-c-c e^{-2 / c}-e^{-2 / c}, \\
g^{\prime \prime}(c) & =-1-e^{-2 / c}-\frac{2}{c} e^{-2 / c}-\frac{2}{c^{2}} e^{-2 / c}<0, \\
g^{\prime \prime \prime}(c) & =-\frac{4}{c^{4}} e^{-2 / c}<0 .
\end{aligned}
$$

We observe that $g($.$) has the same unique critical point as \alpha\left(\right.$. ), i.e. $g^{\prime}(c)=0 \Leftrightarrow c=c_{0}$,

$$
g^{\prime}(c)>0 \Leftrightarrow c<c_{0} \quad \text { and } g^{\prime}(c)<0 \Leftrightarrow c>c_{0} .
$$


As for $\alpha$ we conclude from the strict monotonicity of the second derivatives and from $g^{\prime}\left(c_{0}\right)=$ 0 that

$$
\forall c \in\left(0, c_{0}\right): \quad g\left(c_{0}-c\right)>g\left(c_{0}+c\right) .
$$

We may now prove (25) and take any $\alpha>\alpha_{0}$. As above we choose

$$
0<c_{2}(\alpha)<c_{0}<c_{1}(\alpha) \text { with } \quad \alpha=\alpha\left(c_{1}(\alpha)\right)=\alpha\left(c_{2}(\alpha)\right) .
$$

Inequality (40) yields

$\alpha\left(c_{1}(\alpha)\right)=\alpha\left(c_{2}(\alpha)\right)=\alpha\left(c_{0}-\left(c_{0}-c_{2}(\alpha)\right)\right)>\alpha\left(c_{0}+\left(c_{0}-c_{2}(\alpha)\right)\right)=\alpha\left(2 c_{0}-c_{2}(\alpha)\right)$.

Since $\alpha\left(\right.$. ) is strictly increasing on $\left(c_{0}, \infty\right)$, this yields

$$
c_{1}(\alpha)>2 c_{0}-c_{2}(\alpha) .
$$

On the other hand, $g($.$) is strictly decreasing on \left(c_{0}, \infty\right)$, and we find by means of (41)

$$
g\left(c_{1}(\alpha)\right)<g\left(2 c_{0}-c_{2}(\alpha)\right)=g\left(c_{0}+\left(c_{0}-c_{2}(\alpha)\right)\right)<g\left(c_{0}-\left(c_{0}-c_{2}(\alpha)\right)\right)=g\left(c_{2}(\alpha)\right) \text {. }
$$

Since the Goldschmidt contribution is the same for $c_{1}(\alpha)$ and $c_{2}(\alpha)$, we come up with

$$
\mathscr{A}\left(w_{c_{1}(\alpha)}\right)<\mathscr{A}\left(w_{c_{2}(\alpha)}\right),
$$

as claimed. Finally one should note that $g(c)<0 \Leftrightarrow c>c_{m}$, which shows that in this regime, the "small" catenoid $w_{c_{1}(\alpha)}$ has smaller area than the Goldschmidt solution as well as the "large" catenoid $w_{c_{2}(\alpha)}$.

\section{Appendix B : Divergence form of the Helfrich equation}

We note that the form of $M[u]$ in the case $\varepsilon=0$ is derived in [13, Theorem 3] and is a consequence of the invariance of the Willmore functional with respect to translations. This means that the Helfrich equation can be written in divergence form. For the Willmore equation this was observed and exploited by Rusu [40], Rivière [39] and many others.

Lemma 4 For $u \in C^{4}([-1,1])$ let

$$
M[u](x):=\frac{u(x) u^{\prime}(x) H^{\prime}(x)}{1+u^{\prime}(x)^{2}}+\frac{u(x) H(x)^{2}}{\sqrt{1+u^{\prime}(x)^{2}}}-\frac{H(x)}{1+u^{\prime}(x)^{2}}-\varepsilon \frac{u(x)}{\sqrt{1+u^{\prime}(x)^{2}}} .
$$

Then we have

$$
\begin{aligned}
\frac{\mathrm{d}}{\mathrm{d} x} M[u](x)= & u(x) u^{\prime}(x)\left\{\frac{1}{u(x) \sqrt{1+u^{\prime}(x)^{2}}} \frac{\mathrm{d}}{\mathrm{d} x}\left(\frac{u(x)}{\sqrt{1+u^{\prime}(x)^{2}}} H^{\prime}(x)\right)\right. \\
& \left.+\frac{1}{2} H(x)\left(\frac{u^{\prime \prime}(x)}{\left(1+u^{\prime}(x)^{2}\right)^{3 / 2}}+\frac{1}{u(x) \sqrt{1+u^{\prime}(x)^{2}}}\right)^{2}-2 \varepsilon H(x)\right\} .
\end{aligned}
$$

Proof A straightforward calculation shows that

$$
\begin{aligned}
\frac{\mathrm{d}}{\mathrm{d} x}\left(\frac{u u^{\prime} H^{\prime}}{1+\left(u^{\prime}\right)^{2}}\right) & =\frac{u^{\prime}}{\sqrt{1+\left(u^{\prime}\right)^{2}}} \frac{\mathrm{d}}{\mathrm{d} x}\left(\frac{u H^{\prime}}{\sqrt{1+\left(u^{\prime}\right)^{2}}}\right)+\frac{u u^{\prime \prime} H^{\prime}}{\left(1+\left(u^{\prime}\right)^{2}\right)^{2}} ; \\
\frac{\mathrm{d}}{\mathrm{d} x}\left(\frac{u H^{2}}{\sqrt{1+\left(u^{\prime}\right)^{2}}}\right) & =\frac{u^{\prime} H^{2}}{\sqrt{1+\left(u^{\prime}\right)^{2}}}+2 \frac{u H H^{\prime}}{\sqrt{1+\left(u^{\prime}\right)^{2}}}-\frac{u u^{\prime} u^{\prime \prime} H^{2}}{\left(1+\left(u^{\prime}\right)^{2}\right)^{3 / 2}} ;
\end{aligned}
$$




$$
\begin{aligned}
\frac{\mathrm{d}}{\mathrm{d} x}\left(\frac{H}{1+\left(u^{\prime}\right)^{2}}\right) & =\frac{H^{\prime}}{1+\left(u^{\prime}\right)^{2}}-2 \frac{u^{\prime} u^{\prime \prime} H}{\left(1+\left(u^{\prime}\right)^{2}\right)^{2}} ; \\
\frac{\mathrm{d}}{\mathrm{d} x}\left(\frac{u}{\sqrt{1+\left(u^{\prime}\right)^{2}}}\right) & =2 u u^{\prime} H .
\end{aligned}
$$

Combining the above relations and taking into account that

$$
\frac{u u^{\prime \prime}}{\left(1+\left(u^{\prime}\right)^{2}\right)^{2}}+2 \frac{u H}{\sqrt{1+\left(u^{\prime}\right)^{2}}}-\frac{1}{1+\left(u^{\prime}\right)^{2}}=0
$$

we obtain

$$
\begin{aligned}
& \frac{\mathrm{d}}{\mathrm{d} x} M[u]=u u^{\prime}\left\{\frac{1}{u \sqrt{1+\left(u^{\prime}\right)^{2}}} \frac{\mathrm{d}}{\mathrm{d} x}\left(\frac{u H^{\prime}}{\sqrt{1+\left(u^{\prime}\right)^{2}}}\right)\right. \\
& \left.\quad+H\left(\frac{H}{u \sqrt{1+\left(u^{\prime}\right)^{2}}}-\frac{u^{\prime \prime} H}{\left(1+\left(u^{\prime}\right)^{2}\right)^{3 / 2}}+2 \frac{u^{\prime \prime}}{u\left(1+\left(u^{\prime}\right)^{2}\right)^{2}}\right)-2 \varepsilon H\right\} \\
& =u u^{\prime}\left\{\frac{1}{u \sqrt{1+\left(u^{\prime}\right)^{2}}} \frac{\mathrm{d}}{\mathrm{d} x}\left(\frac{u H^{\prime}}{\sqrt{1+\left(u^{\prime}\right)^{2}}}\right)+\frac{1}{2} H\left(\frac{u^{\prime \prime}}{\left(1+\left(u^{\prime}\right)^{2}\right)^{3 / 2}}+\frac{1}{u \sqrt{1+\left(u^{\prime}\right)^{2}}}\right)^{2}-2 \varepsilon H\right\} .
\end{aligned}
$$

\section{Appendix C: Estimating the oscillations}

In order to prove the auxiliary results used in Sect. 4 we will give a description of the oscillatory behaviour of the functions

$$
\begin{aligned}
& x \mapsto A \cosh (a x) \cos (a x)-B \sinh (a x) \sin (a x), \\
& x \mapsto A \sinh (a x) \cos (a x)-B \cosh (a x) \sin (a x),
\end{aligned}
$$

where $A, B$ and $a$ are given constants.

Lemma 5 Let $K \neq 0$ be a given constant and consider the function

$$
\eta:[0,1) \rightarrow[0, \infty), \quad \eta(y):=1-\frac{\left(1+K^{2}\right) y^{2}}{1+K^{2} y^{2}} .
$$

Then for all $y \in[0,1)$ we have $\eta(y)>0$. Furthermore:

a) If $K<-1$, then there exists $y_{*} \in\left(0, \frac{1}{\sqrt{2}}\right)$ such that $1+K \eta\left(y_{*}\right)=0$ and $1+K \eta(y)<0$ on $\left(0, y_{*}\right), 1+K \eta(y)>0$ on $\left(y_{*}, 1\right)$.

b) If $K \geq-1$, then $1+K \eta(y)>0$ for all $y \in(0,1)$.

Proof Rewriting

$$
\eta(y)=\frac{1-y^{2}}{1+K^{2} y^{2}}=\frac{1+K^{2}}{K^{2}} \cdot \frac{1}{1+K^{2} y^{2}}-\frac{1}{K^{2}}
$$

shows that $\eta($.$) is strictly decreasing and hence that \eta(y)>0$ for $y \in[0,1)$. If $K<-1$ a calculation shows that

$$
1+K \eta(y)=\frac{K+1}{1+K^{2} y^{2}}\left(1+\frac{K-1}{1+\frac{1}{K}} y^{2}\right),
$$


which implies (a) with $y_{*}=\left(\frac{1+\frac{1}{K}}{1-K}\right)^{\frac{1}{2}}$. Note that $y_{*} \in\left(0, \frac{1}{\sqrt{2}}\right)$, since $K<-1$. The assertion (b) is straightforward.

Proposition 1 Consider constants $a \neq 0$ and $A, B$ which do not vanish simultaneously, i.e. $A \neq 0$ or $B \neq 0$. Define the even function

$$
h: \mathbb{R} \rightarrow \mathbb{R}, \quad h(x):=A \cosh (a x) \cos (a x)-B \sinh (a x) \sin (a x) .
$$

Then the local extrema of $h$ are isolated and strictly increasing in their absolute values on the positive real axis, i.e.

$$
\left|h\left(x_{1}\right)\right|<\left|h\left(x_{2}\right)\right| \quad \text { for local extrema } 0 \leq x_{1}<x_{2} .
$$

Proof Without loss of generality we assume that $a=1$ since $h$ is symmetric and the statement is invariant under linear transformations. Since the case where $A=0$ or $B=0$ is quite simple we may assume that $A \neq 0$ and $B \neq 0$. Further we can restrict ourselves to the case $A>0$; otherwise $-h$ is considered. Setting $A=1$ would also be admissible but we like to keep the " $A$ " to highlight the connection to $B$ in the following discussion. Because $h^{\prime \prime}(x)=-2 A \sinh (x) \sin (x)-2 B \cosh (x) \cos (x)$ we see that $h$ has also a strict local minimum in 0 iff $B<0$ and a strict local maximum in 0 iff $B \geq 0$. In the first case $h$ must have a further extremum before its first zero.

First of all we rewrite

$$
h(x)=A \cosh (x) \cos (x)-B \sinh (x) \sin (x)=E(x) \cos (x+\varphi(x)),
$$

with

$$
E(x):=\left[A^{2} \cosh ^{2}(x)+B^{2} \sinh ^{2}(x)\right]^{\frac{1}{2}}
$$

and $\varphi$ is the smooth angular map defined modulo $2 \pi$ by

$$
\cos (\varphi(x))=\frac{A}{E(x)} \cosh (x) \quad \text { and } \quad \sin (\varphi(x))=\frac{B}{E(x)} \sinh (x) .
$$

We choose $\varphi$ such that it is uniquely determined by $\varphi(0)=0$, i.e. $\varphi(x) \in(-\pi / 2, \pi / 2)$. If $B>0$ then $\varphi(x) \in[0, \pi / 2)$, if $B<0$ then $\varphi(x) \in(-\pi / 2,0]$, and if $B=0$, then $\varphi(x) \equiv 0$. For the derivative of $\varphi$ we compute

$$
\begin{aligned}
\cos (\varphi(x)) \varphi^{\prime}(x) & =\frac{\mathrm{d}}{\mathrm{d} x}(\sin (\varphi(x)))=\frac{B}{E(x)} \cosh (x)-\frac{B}{E(x)^{2}} \sinh (x) E^{\prime}(x) \\
& =\frac{B}{E(x)} \cosh (x)-\frac{B\left(A^{2}+B^{2}\right)}{E(x)^{3}} \sinh ^{2}(x) \cosh (x)
\end{aligned}
$$

which yields

$$
\varphi^{\prime}(x)=\frac{B}{A}\left[1-\frac{\left(A^{2}+B^{2}\right) \sinh ^{2}(x)}{A^{2} \cosh ^{2}(x)+B^{2} \sinh ^{2}(x)}\right]=\frac{B}{A}\left[1-\frac{\left(1+\frac{B^{2}}{A^{2}}\right) \tanh ^{2}(x)}{1+\frac{B^{2}}{A^{2}} \tanh ^{2}(x)}\right] .
$$

Putting $K=\frac{B}{A}$ and $y=\tanh (x)$ we apply Lemma 5 to $\eta(y):=\frac{1}{K} \varphi^{\prime}(x)$. Observing that $\frac{\mathrm{d}}{\mathrm{d} x}(x+\varphi(x))=1+K \eta(y)$ we see that $\frac{\mathrm{d}}{\mathrm{d} x}(x+\varphi(x))$ is either always positive or negative first and then positive. Because $x+\varphi(x)>-\frac{\pi}{2}$, Id $+\varphi$ has to become strictly increasing before the first zero of $h$. Denoting $0<x_{1}<\ldots<x_{k}<\ldots$ the zeroes of $h$ this shows:

$$
x_{k}+\varphi\left(x_{k}\right)=\left(k-\frac{1}{2}\right) \pi \text {. }
$$


In particular

$$
x_{k} \in \begin{cases}\left((k-1) \pi,\left(k-\frac{1}{2}\right) \pi\right) & \text { if } B>0, \\ {\left[\left(k-\frac{1}{2}\right) \pi, k \pi\right)} & \text { if } B \leq 0 .\end{cases}
$$

Moreover, $|\cos (x+\varphi(x))|$ attains in each interval $\left(x_{k}, x_{k+1}\right)$ the value 1 .

Next we also rewrite the derivative of $h$

$$
h^{\prime}(x)=(A-B) \sinh (x) \cos (x)-(A+B) \cosh (x) \sin (x)=F(x) \cos (x+\psi(x)),
$$

with

$$
F(x):=\left[(A+B)^{2} \cosh ^{2}(x)+(A-B)^{2} \sinh ^{2}(x)\right]^{\frac{1}{2}}
$$

and the smooth function $\psi$ determined by

$$
\cos (\psi(x))=\frac{A-B}{F(x)} \sinh (x) \quad \text { and } \quad \sin (\psi(x))=\frac{A+B}{F(x)} \cosh (x) .
$$

Here we choose $\psi(0)=\frac{\pi}{2}$ in case $-A<B, \psi(0)=0$ if $-B=A$ and $\psi(0)=-\frac{\pi}{2}$ else, such that $\psi(x) \in(-\pi, \pi)$. Similar to $\varphi$ we have

$$
\begin{aligned}
\psi^{\prime}(x) & =-\frac{A-B}{A+B}\left[1-\frac{\left((A+B)^{2}+(A-B)^{2}\right) \sinh ^{2}(x)}{(A+B)^{2} \cosh ^{2}(x)+(A-B)^{2} \sinh ^{2}(x)}\right] \\
& =\frac{B-A}{A+B}\left[1-\frac{\left(1+\frac{(B-A)^{2}}{(A+B)^{2}}\right) \tanh ^{2}(x)}{1+\frac{(B-A)^{2}}{(A+B)^{2}} \tanh ^{2}(x)}\right] .
\end{aligned}
$$

Let $0=y_{0}<y_{1}<\ldots<y_{k}<\ldots$ denote the zeroes of $h^{\prime}$.

Now, since $E$ and $F$ are strictly increasing, the proposition follows if we are able to show the claim:

Between two consecutive zeroes of $h$ in $(0, \infty)$ there is exactly one zero of $h^{\prime}$ and vice versa.

Claims (C) and (D) yield the proof of the proposition, because:

- Case $B \geq 0,0$ is a local maximum of $h$ :

We shall see that $0=y_{0}<x_{1}<\ldots<y_{k}<x_{k+1}<y_{k+1}<\ldots$ and the above claims yield:

$$
\begin{aligned}
\sup _{x \in\left[x_{k-1}, x_{k}\right]}|h(x)| & =\left|h\left(y_{k-1}\right)\right|<\sup _{x \in\left[x_{k-1}, x_{k}\right]} E(x) \leq \inf _{x \in\left[x_{k}, x_{k+1}\right]} E(x)<\left|h\left(y_{k}\right)\right| \\
& =\sup _{x \in\left[x_{k}, x_{k+1}\right]}|h(x)| .
\end{aligned}
$$

The strict inequalities above are due to the strict monotonicity of $E$ and to the facts that $h\left(x_{k}\right)=0$ and that $|\cos ()|=$.1 once on each $\left[x_{k}, x_{k+1}\right]$.

- Case $B<0,0$ is a local minimum of $h$ : We shall see that $0=y_{0}<y_{1}<x_{1}<\ldots<$ $y_{k}<x_{k}<y_{k+1}<\ldots$ and the above claims yield:

$$
\begin{aligned}
\sup _{x \in\left[x_{k-1}, x_{k}\right]}|h(x)| & =\left|h\left(y_{k}\right)\right|<\sup _{x \in\left[x_{k-1}, x_{k}\right]} E(x) \leq \inf _{x \in\left[x_{k}, x_{k+1}\right]} E(x)<\left|h\left(y_{k+1}\right)\right| \\
& =\sup _{x \in\left[x_{k}, x_{k+1}\right]}|h(x)| .
\end{aligned}
$$




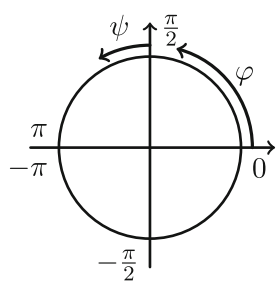

case: $0<A<B$

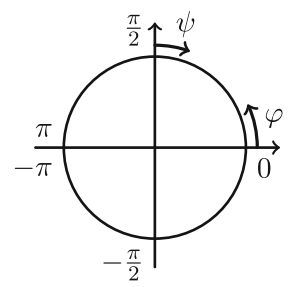

case: $0<B<A$

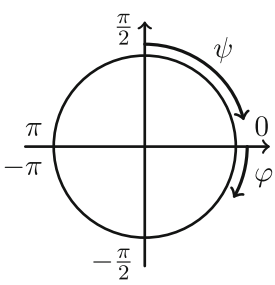

case: $-A<B<0$

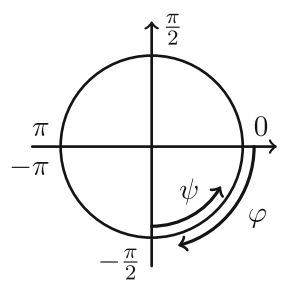

case: $B<-A<0$

Fig. 5 Sketch of the qualitative behaviour of $\varphi(x)$ and $\psi(x)$ as $x$ is increased in four different cases. Here the cases $B=A, B=0$ and $B=-A$ are omitted

Note that the local extremum at $x=0$ has always the smallest absolute value among all local extrema.

To prove the claim we need to discuss several cases (see Fig. 5):

Case $0<A \leq B$ : Combining (42) and (43) with Lemma 5 by setting $K=\frac{B}{A}$, resp. $K=\frac{B-A}{A+B}$, and $y=\tanh (x)$ we see that $\varphi$ and $\psi$ are strictly increasing with

$$
\begin{aligned}
& \varphi(0)=0, \quad \varphi(\infty):=\lim _{x \rightarrow \infty} \varphi(x)=\arcsin \left(\frac{B}{\sqrt{A^{2}+B^{2}}}\right) \in\left(0, \frac{\pi}{2}\right), \\
& \psi(0)=\frac{\pi}{2}, \quad \psi(\infty):=\lim _{x \rightarrow \infty} \psi(x)=\arccos \left(\frac{A-B}{\sqrt{(A+B)^{2}+(A-B)^{2}}}\right) \in\left[\frac{\pi}{2}, \pi\right) .
\end{aligned}
$$

This immediately implies for all $x>0$

$$
\varphi(x)<\psi(x)<\varphi(x)+\pi, \quad \text { i. e. } \quad x+\varphi(x)<x+\psi(x)<x+\varphi(x)+\pi .
$$

Since Id $+\psi$ is strictly increasing we have that $y_{k}+\psi\left(y_{k}\right)=(k+1 / 2) \pi$ and further

$$
\begin{aligned}
x_{k}+\psi\left(x_{k}\right) & <x_{k}+\varphi\left(x_{k}\right)+\pi=\left(k+\frac{1}{2}\right) \pi \\
& =y_{k}+\psi\left(y_{k}\right)=x_{k+1}+\varphi\left(x_{k+1}\right)<x_{k+1}+\psi\left(x_{k+1}\right) .
\end{aligned}
$$

Hence, again by strict monotonicity of Id $+\psi$

$$
x_{k}<y_{k}<x_{k+1},
$$

which proves Claim (D).

Case $0 \leq B<A$ : Applying Lemma 5 once again we see that $\varphi$ is still strictly increasing, but $\psi$ is strictly decreasing with

$$
\begin{aligned}
& \varphi(0)=0, \quad \varphi(\infty) \in\left[0, \frac{\pi}{2}\right), \\
& \psi(0)=\frac{\pi}{2}, \quad \psi(\infty)=\arcsin \left(\frac{A+B}{\sqrt{(A+B)^{2}+(A-B)^{2}}}\right) \in\left(0, \frac{\pi}{2}\right) .
\end{aligned}
$$

This implies for all $x>0$ that $|\psi(x)-\varphi(x)|<\frac{\pi}{2}$. Moreover, since $B<A$

$$
\varphi(\infty)=\arcsin \left(\frac{B}{\sqrt{A^{2}+B^{2}}}\right)<\arcsin \left(\frac{A+B}{\sqrt{(A+B)^{2}+(A-B)^{2}}}\right)=\psi(\infty),
$$


and (44) still holds. Obviously Id $+\varphi$ is strictly increasing. But as $\frac{B-A}{A+B} \geq-1$ we also get from Lemma 5 that $\mathrm{Id}+\psi$ is strictly increasing so that $y_{k}+\psi\left(y_{k}\right)=(k+1 / 2) \pi$ as above. Like in the previous case we obtain again the validity of Claim (D).

Case $-A<B<0: \varphi$ and $\psi$ are strictly decreasing with

$$
\varphi(0)=0, \quad \varphi(\infty) \in\left(-\frac{\pi}{2}, 0\right), \quad \psi(0)=\frac{\pi}{2}, \quad \psi(\infty) \in\left(0, \frac{\pi}{2}\right) .
$$

Similar to the first case this immediately implies (44). The strict monotonicity of Id $+\varphi$ follows from Lemma 5 like in the previous step as $\frac{B}{A}>-1$. From Lemma 5 with $\frac{B-A}{A+B}<-1$ we also know that Id $+\psi$ possesses a zero at $x_{*}<\tanh ^{-1}(1 / \sqrt{2}) \approx 0.8814$ so that for $x>x_{*}$ it is strictly increasing and decreasing for $x \in\left(0, x_{*}\right)$. In the latter situation we have

$$
-\frac{\pi}{2}<0+\varphi(0)<x+\varphi(x)<x_{*}+\varphi\left(x_{*}\right)<x_{*}+\psi\left(x_{*}\right) \leq x+\psi(x)<0+\psi(0)=\frac{\pi}{2},
$$

so that neither $h$ nor $h^{\prime}$ have a zero in $\left(0, x_{*}\right]$. Hence $y_{1}>x_{*}$ and

$$
y_{0}+\psi\left(y_{0}\right)=\frac{\pi}{2}, \quad \forall k \in \mathbb{N}: \quad y_{k}+\psi\left(y_{k}\right)=\left(k-\frac{1}{2}\right) \pi .
$$

For $k \in \mathbb{N}$ we obtain using the monotonicity behaviour of $\operatorname{Id}+\varphi$ and $\operatorname{Id}+\psi$ :

$$
\begin{gathered}
y_{k}+\varphi\left(y_{k}\right)<y_{k}+\psi\left(y_{k}\right)=\left(k-\frac{1}{2}\right) \pi=x_{k}+\varphi\left(x_{k}\right) \Rightarrow y_{k}<x_{k}, \\
y_{k+1}+\psi\left(y_{k+1}\right)=\left(k+\frac{1}{2}\right) \pi=x_{k}+\varphi\left(x_{k}\right)+\pi>x_{k}+\psi\left(x_{k}\right) \Rightarrow x_{k}<y_{k+1} .
\end{gathered}
$$

We conclude that $0=y_{0}<y_{1}<x_{1}<\ldots<y_{k}<x_{k}<y_{k+1}<\ldots$, which is again Claim (D).

Case $B \leq-A<0$ : We only prove the claim for $B<-A$. The case $B=-A$ follows from a similar discussion. Here $\varphi$ is strictly decreasing and $\psi$ is strictly increasing with

$$
\varphi(0)=0, \quad \varphi(\infty) \in\left(-\frac{\pi}{2}, 0\right), \quad \psi(0)=-\frac{\pi}{2}, \quad \psi(\infty) \in\left(-\frac{\pi}{2}, 0\right) .
$$

We first would like to establish the separation property (44). Obviously $|\varphi(x)-\psi(x)|<\frac{\pi}{2}$ holds for all $x>0$. Unfortunately $\varphi$ and $\psi$ will intersect in a point $x_{*}>0$ so that (44) will only hold for $x>x_{*}$. For $x>0$ we consider the "distance" between $\varphi$ and $\psi$ given by

$$
\begin{aligned}
G(x)^{2}:= & {[\cos (\varphi(x))-\cos (\psi(x))]^{2}+[\sin (\varphi(x))-\sin (\psi(x))]^{2} } \\
= & \cos ^{2}(\varphi(x))-2 \cos (\varphi(x)) \cos (\psi(x))+\cos ^{2}(\psi(x)) \\
& +\sin ^{2}(\varphi(x))-2 \sin (\varphi(x)) \sin (\psi(x))+\sin ^{2}(\psi(x)) \\
= & 2-2 \cos (\varphi(x)) \cos (\psi(x))-2 \sin (\varphi(x)) \sin (\psi(x)) \\
= & 2-\frac{2 A(A-B)}{E(x) F(x)} \cosh (x) \sinh (x)-\frac{2 B(A+B)}{E(x) F(x)} \sinh (x) \cosh (x) \\
= & 2-2 \frac{\left(A^{2}+B^{2}\right) \cosh (x) \sinh (x)}{\left[A^{2} \cosh ^{2}(x)+B^{2} \sinh ^{2}(x)\right]^{1 / 2}\left[(A+B)^{2} \cosh ^{2}(x)+(A-B)^{2} \sinh ^{2}(x)\right]^{1 / 2}} \\
= & 2-2 \frac{\left(A^{2}+B^{2}\right) \tanh (x)}{\left[A^{2}+B^{2} \tanh ^{2}(x)\right]^{1 / 2}\left[(A+B)^{2}+(A-B)^{2} \tanh ^{2}(x)\right]^{1 / 2}} .
\end{aligned}
$$


If we substitute $y=\tanh (x)$ we find that $G(x)^{2}=0$ if

$$
(A-B)^{2} B^{2} y^{4}-2 A B\left(A^{2}-B^{2}\right) y^{2}+A^{2}(A+B)^{2}=0,
$$

whose positive solution is given by $y_{*}=\left(\frac{A(A+B)}{B(A-B)}\right)^{\frac{1}{2}}$. Recalling that $B<-A<0$ we find

$$
y_{*}=\left(\frac{A(|B|-A)}{|B|(A+|B|)}\right)^{\frac{1}{2}}<\left(\frac{A|B|}{|B|(A+|B|)}\right)^{\frac{1}{2}}<\frac{1}{\sqrt{2}} \text {. }
$$

Thus $\varphi$ and $\psi$ will intersect in the point $x_{*}=\tanh ^{-1}\left(y_{*}\right)<\tanh ^{-1}(1 / \sqrt{2}) \approx 0.8814$. From the strict monotonicity of $\varphi$ and $\psi$ we then get that $\psi(x)<\varphi(x)$ for $x<x_{*}$ and $\psi(x)>\varphi(x)$ for $x>x_{*}$. Since $\psi<0$ and $\varphi<0$ we find that $x_{1}>\frac{\pi}{2}, y_{1}>\frac{\pi}{2}$ and

$$
\forall k \in \mathbb{N}_{0}: \quad y_{k}+\psi\left(y_{k}\right)=\left(k-\frac{1}{2}\right) \pi .
$$

As in the previous case we see that $\mathrm{Id}+\varphi$ is strictly increasing on $[\pi / 2, \infty)$ and $\mathrm{Id}+\psi$ on $[0, \infty)$. This yields for $k \in \mathbb{N}$ :

$$
\begin{gathered}
y_{k}+\psi\left(y_{k}\right)=\left(k-\frac{1}{2}\right) \pi=x_{k}+\varphi\left(x_{k}\right)<x_{k}+\psi\left(x_{k}\right) \Rightarrow y_{k}<x_{k}, \\
y_{k+1}+\psi\left(y_{k+1}\right)=\left(k+\frac{1}{2}\right) \pi=x_{k}+\varphi\left(x_{k}\right)+\pi>x_{k}+\psi\left(x_{k}\right) \Rightarrow x_{k}<y_{k+1} .
\end{gathered}
$$

We conclude that $0=y_{0}<y_{1}<x_{1}<\ldots<y_{k}<x_{k}<y_{k+1}<\ldots$, so that again Claim (D) holds true.

Remark 3 The proof of Proposition 1 also shows, that we always have

$$
\lim _{x \rightarrow \infty}(\psi(x)-\varphi(x))=\frac{\pi}{4} .
$$

In fact, recalling the function $G$ in (45) we obtain

$$
\lim _{x \rightarrow \infty} G(x)=2-2 \frac{A^{2}+B^{2}}{\left[A^{2}+B^{2}\right]^{1 / 2}\left[2 A^{2}+2 B^{2}\right]^{1 / 2}}=2-\sqrt{2}
$$

which shows that the points $\exp (i \psi(\infty))$ and $\exp (i \varphi(\infty))$ on the unit circle are $\sqrt{2-\sqrt{2}}$ apart from each other. The claim now follows from $2 \arcsin (\sqrt{2-\sqrt{2}} / 2)=\pi / 4$.

Corollary 4 Let $a>0$ be given. Then the function

$$
\begin{aligned}
{[0,1] \ni x \mapsto } & {[\sinh (a) \cos (a)+\cosh (a) \sin (a)] \cosh (a x) \cos (a x) } \\
& -[\sinh (a) \cos (a)-\cosh (a) \sin (a)] \sinh (a x) \sin (a x)
\end{aligned}
$$

attains its maximum at $x=1$.

Proof Here we apply Proposition 1 with

$$
\begin{aligned}
& A:=\sinh (a) \cos (a)+\cosh (a) \sin (a), \\
& B:=\sinh (a) \cos (a)-\cosh (a) \sin (a) .
\end{aligned}
$$


Note that we can exclude the case $A=B=0$. Otherwise we would have $\cos (a)=\sin (a)=0$ which is not possible. Let $h$ denote the function from Proposition 1 with our choice for $A, B$ and $a$. In what follows we show, that $x=1$ is a local maximum of $h$. Therefore we compute

$$
\begin{aligned}
h(x)= & A \cosh (a x) \cos (a x)-B \sinh (a x) \sin (a x) \\
= & {[\sinh (a) \cos (a)+\cosh (a) \sin (a)] \cosh (a x) \cos (a x) } \\
& -[\sinh (a) \cos (a)-\cosh (a) \sin (a)] \sinh (a x) \sin (a x), \\
\frac{1}{a} h^{\prime}(x)= & (A-B) \sinh (a x) \cos (a x)-(A+B) \cosh (a x) \sin (a x) \\
= & 2 \cosh (a) \sin (a) \sinh (a x) \cos (a x)-2 \sinh (a) \cos (a) \cosh (a x) \sin (a x), \\
\frac{1}{a^{2}} h^{\prime \prime}(x)= & -2 A \sinh (a x) \sin (a x)-2 B \cosh (a x) \cos (a x) \\
= & -2[\sinh (a) \cos (a)+\cosh (a) \sin (a)] \sinh (a x) \sin (a x) \\
& -2[\sinh (a) \cos (a)-\cosh (a) \sin (a)] \cosh (a x) \cos (a x) .
\end{aligned}
$$

We conclude that

$$
\begin{aligned}
h(1)= & \sinh (a) \cosh (a) \cos ^{2}(a)+\cosh ^{2}(a) \sin (a) \cos (a) \\
& -\sinh ^{2}(a) \sin (a) \cos (a)+\sinh (a) \cosh (a) \sin ^{2}(a) \\
= & \sinh (a) \cosh (a)+\sin (a) \cos (a) \\
> & 0, \\
\frac{1}{a} h^{\prime}(1)= & 2 \cosh (a) \sin (a) \sinh (a) \cos (a)-2 \sinh (a) \cos (a) \cosh (a) \sin (a) \\
= & 0, \\
\frac{1}{a^{2}} h^{\prime \prime}(1)= & -2 \sinh ^{2}(a) \sin (a) \cos (a)-2 \sinh (a) \cosh (a) \sin ^{2}(a) \\
& -2 \sinh ^{2}(a) \cosh (a) \cos ^{2}(a)+2 \cosh (a) \sin (a) \cos (a) \\
= & -2 \sinh (a) \cosh (a)+2 \sin (a) \cos (a) \\
< & 0 .
\end{aligned}
$$

From Proposition 1 we conclude, that $h(1)$ as a local maximum satisfies

$$
h(1)=|h(1)|>|h(x)| \geq h(x),
$$

where $x \in[0,1)$ is an arbitrary local extremum. In particular this must also hold not only for $x$ being a local extremum, but for any arbitrary point in $[0,1)$ which proves the claim.

Lemma 6 We consider the function

$$
f: D_{f}:=[0, \infty) \backslash \pi \mathbb{N} \rightarrow \mathbb{R}, \quad f(x):=\frac{\tanh (x)}{\tan (x)} .
$$

This function is strictly decreasing on each connected component of its domain of definition,

$$
\forall x \in D_{f} \backslash\{0\}: f^{\prime}(x)<0 .
$$

Since $f(0)=1$ and $\lim _{x \rightarrow k \pi \pm 0} f(x)= \pm \infty$ there exists a smallest strictly positive solution $a_{c} \in\left(\pi, \frac{3}{2} \pi\right)$ of the equation $f(x)=1$, i.e.

$$
\tan \left(a_{c}\right)=\tanh \left(a_{c}\right), \quad \forall x \in(0, \pi): \frac{\tanh (x)}{\tan (x)}<1, \quad \forall x \in\left(\pi, a_{c}\right): \frac{\tanh (x)}{\tan (x)}>1 .
$$




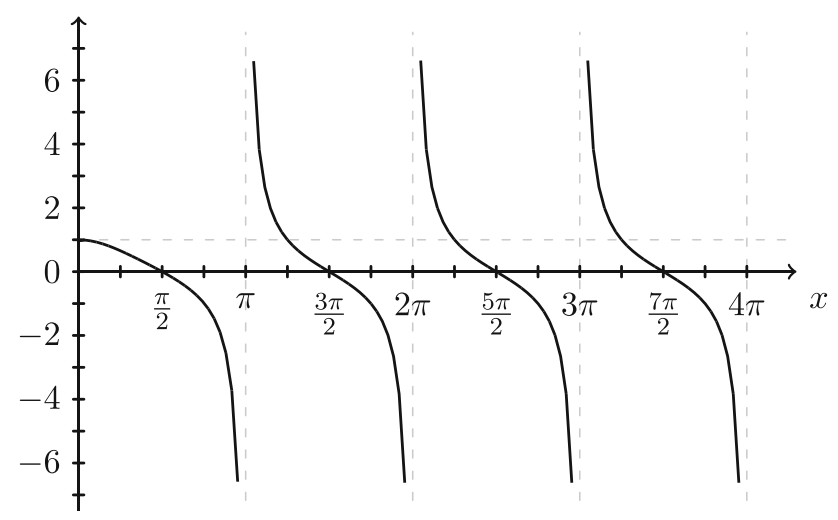

Fig. 6 Graph of $x \mapsto \frac{\tanh (x)}{\tan (x)}$

Proof We calculate for $x \in D_{f} \backslash\{0\}$ :

$$
\begin{aligned}
& f(x)=\frac{\sinh (x) \cos (x)}{\cosh (x) \sin (x)} \\
& f^{\prime}(x)=\frac{(\cosh (x) \cos (x)-\sinh (x) \sin (x)) \cdot \cosh (x) \sin (x)-\sinh (x) \cos (x) \cdot(\sinh (x) \sin (x)+\cosh (x) \cos (x))}{\cosh ^{2}(x) \sin ^{2}(x)} \\
& =\frac{\sin (x) \cos (x) \cdot\left(\cosh ^{2}(x)-\sinh ^{2}(x)\right)-\sinh (x) \cosh (x) \cdot\left(\sin ^{2}(x)+\cos ^{2}(x)\right)}{\cosh ^{2}(x) \sin ^{2}(x)} \\
& =\frac{\sin (x) \cos (x)-\sinh (x) \cosh (x)}{\cosh ^{2}(x) \sin ^{2}(x)}=\frac{\sin (2 x)-\sinh (2 x)}{2 \cosh ^{2}(x) \sin ^{2}(x)}<0
\end{aligned}
$$

Lemma 7 Let $\left.a_{c} \in\left(\pi, \frac{3}{2} \pi\right)\right)$ be as in Lemma 6. Then for a $>0$ we have that $\forall x \in(0, a): \quad \cosh (a) \sin (a) \sinh (x) \cos (x)-\sinh (a) \cos (a) \cosh (x) \sin (x)>0$,

iff

$$
a \in\left(0, a_{c}\right]
$$

Proof The claim is obvious for $a \in \pi \mathbb{N}$, so we only need to consider $a \in(0, \infty) \backslash \pi \mathbb{N}$. We consider the function $f$ as in Lemma 6.

Case $a \in(0, \pi)$ :

Since $f$ is strictly decreasing on $(0, a)$, we have for all $x \in(0, a)$ :

$$
\begin{aligned}
f(x) & >f(a) \\
\Rightarrow \quad \frac{\tanh (x)}{\tan (x)} & >\frac{\tanh (a)}{\tan (a)} \\
\Rightarrow \quad \sinh (x) \cos (x) \cosh (a) \sin (a) & >\sinh (a) \cos (a) \cosh (x) \sin (x) .
\end{aligned}
$$

In the last step we used that $\sin (x)>0, \sin (a)>0$.

Case $a \in\left(\pi, a_{c}\right]$ :

Here $f(a) \geq f\left(a_{c}\right)=1$. We consider first $x \in(\pi, a)$. Thanks to Lemma 6 we start with

$$
f(x)>f(a) \Rightarrow \frac{\tanh (x)}{\tan (x)}>\frac{\tanh (a)}{\tan (a)} \text {. }
$$


Since in this case $\sin (x)<0, \sin (a)<0$, we end up again with (46). For $x \in(0, \pi)$, the starting point is

$$
f(x)<1=f\left(a_{c}\right) \leq f(a) \Rightarrow \frac{\tanh (x)}{\tan (x)}<\frac{\tanh (a)}{\tan (a)} .
$$

But since now $\sin (x)>0, \sin (a)<0,(46)$ follows again. For $x=\pi$, the claim is obvious. Case $a>a_{c}$ :

According to Lemma 6 and the definition of $a_{c}, f(0, a)=\mathbb{R}$ so that we always find $x_{1}, x_{2} \in$ $(0, a)$ with

$$
\frac{\tanh \left(x_{1}\right)}{\tan \left(x_{1}\right)}>\frac{\tanh (a)}{\tan (a)}>\frac{\tanh \left(x_{2}\right)}{\tan \left(x_{2}\right)}
$$

and both $x_{1}, x_{2}$ in the same interval $(k \pi,(k+1) \pi)$. So the $\operatorname{sign}$ of $\sin \left(x_{1}\right)$ and $\sin \left(x_{2}\right)$ coincides. Depending on the sign of $\sin (a)$ one chooses $x_{0}=x_{1}$ or $x_{0}=x_{2}$ respectively and ends up in each case with a point $x_{0} \in(0, a)$ for which (46) is violated, while in the other point (46) is satisfied.

\section{References}

1. Barrett, J.W., Garcke, H., Nürnberg, R.: Stable variational approximations of boundary value problems for Willmore flow with Gaussian curvature. IMA J. Numer. Anal. 37(4), 1657-1709 (2017)

2. Bauer, M., Kuwert, E.: Existence of minimizing Willmore surfaces of prescribed genus. Int. Math. Res. Not. 2003(10), 553-576 (2003)

3. Bergner, M., Dall'Acqua, A., Fröhlich, S.: Willmore surfaces of revolution with two prescribed boundary circles. J. Geom. Anal. 23(1), 283-302 (2013)

4. Bergner, M., Jakob, R.: Sufficient conditions for Willmore immersions in $\mathbb{R}^{3}$ to be minimal surfaces and Erratum. Ann. Glob. Anal. Geom. 45(2), 129-146 (2014)

5. Bernard, Y., Wheeler, G., Wheeler, V.-M.: Rigidity and stability of spheres in the Helfrich model. Interfaces Free Bound. 19(4), 495-523 (2017)

6. Brazda, K., Lussardi, L., Stefanelli, U.: Existence of varifold minimizers for the multiphase CanhamHelfrich functional. Calc. Var. Partial Differ. Equ. 59, article no. 93 (2020)

7. Bryant, R., Griffiths, P.: Reduction for constrained variational problems and $\int \frac{1}{2} k^{2} \mathrm{~d} s$. Am. J. Math. 108(3), 525-570 (1986)

8. Canham, P.B.: The minimum energy of bending as a possible explanation of the biconcave shape of the human red blood cell. J. Theor. Biol. 26(1), 61-76 (1970)

9. Choksi, R., Morandotti, M., Veneroni, M.: Global minimizers for axisymmetric multiphase membranes. ESAIM Eur. Ser. Appl. Ind. Math COCV Control Optim. Calc. Var. 19(4), 1014-1029 (2013)

10. Choksi, R., Veneroni, M.: Global minimizers for the doubly-constrained Helfrich energy: the axisymmetric case. Calc. Var. Partial. Differ. Equ. 48(3-4), 337-366 (2013)

11. Da Lio, F., Palmurella, F., Rivière, T.: A resolution of the Poisson problem for elastic plates. Arch. Ration. Mech. Anal. 236(3), 1593-1676 (2020)

12. Dall'Acqua, A., Deckelnick, K., Grunau, H-Ch.: Classical solutions to the Dirichlet problem for Willmore surfaces of revolution. Adv. Calc. Var. 1(4), 379-397 (2008)

13. Dall'Acqua, A., Deckelnick, K., Wheeler, G.: Unstable Willmore surfaces of revolution subject to natural boundary conditions. Calc. Var. Partial Differ. Equ. 48(3-4), 293-313 (2013)

14. Dall'Acqua, A., Fröhlich, S., Grunau, H-Ch., Schieweck, F.: Symmetric Willmore surfaces of revolution satisfying arbitrary Dirichlet boundary data. Adv. Calc. Var. 4(1), 1-81 (2011)

15. Deckelnick, K., Grunau, H-Ch.: A Navier boundary value problem for Willmore surfaces of revolution. Analysis 29, 229-258 (2009)

16. Deckelnick, K., Grunau, H-Ch., Röger, M.: Minimising a relaxed Willmore functional for graphs subject to boundary conditions. Interfaces Free Bound. 19(1), 109-140 (2017)

17. Deimling, K.: Nonlinear Funct. Anal. Springer-Verlag, Berlin etc (1985)

18. Doemeland, M.: Axialsymmetrische Minimierer des Helfrich-Funktionals, Master's thesis, Otto-vonGuericke-Universität Magdeburg (2017), available online at https://www.math.ovgu.de/grunau.html

19. Eichmann, S.: The Helfrich boundary value problem. Calc. Var. 58(1), 34 (2019) 
20. Eichmann, S., Grunau, H-Ch.: Existence for Willmore surfaces of revolution satisfying non-symmetric Dirichlet boundary conditions. Adv. Calc. Var. 12, 333-361 (2019)

21. Eichmann, S., Koeller, A.: Symmetry for Willmore surfaces of revolution. J. Geom. Anal. 27(1), 618-642 (2017)

22. Euler, L.: Opera Omnia, Ser. 1, 24, Zürich: Orell Füssli (1952)

23. Gazzola, F., Grunau, H-Ch., Sweers, G.: Polyharmonic Boundary Value Problems, Lecture Notes in Mathematics 1991, Springer-Verlag, Berlin (2010)

24. Germain, S.: Recherches sur la théorie des surfaces élastiques. Mme. Ve. Courcier (1821)

25. Giaquinta, M., Hildebrandt, S.: Calculus of Variations, Vol. I and II. Grundlehren der mathematischen Wissenschaften 310, 311, Springer-Verlag, Berlin etc. (2004)

26. Grunau, H.-Ch.: The asymptotic shape of a boundary layer of symmetric Willmore surfaces of revolution. In: Inequalities and applications 2010, Internat. Ser. Numer. Math.161, 19-29, Birkhäuser/Springer, Basel (2012)

27. Helfrich, W.: Elastic properties of lipid bilayers: Theory and possible experiments. Z. Naturforsch. Teil C 28(11), 693-703 (1973)

28. Kuwert, E., Schätzle, R.: Gradient flow for the Willmore functional. Commun. Anal. Geom. 10(2), 307339 (2002)

29. Kuwert, E., Schätzle, R.: Removability of point singularities of Willmore surfaces. Ann. Math. (2) 160(1), 315-357 (2004)

30. Kuwert, E., Schätzle, R.: The Willmore functional. In: Topics in modern regularity theory, vol. 13 of CRM Series, pp. 1-115. Ed. Norm., Pisa (2012)

31. Mandel, R.: Explicit formulas, symmetry and symmetry breaking for Willmore surfaces of revolution. Ann. Glob. Anal. Geom. 54(2), 187-236 (2018)

32. Marques, F.C., Neves, A.: The Willmore Conjecture. Jahresber. Dtsch. Math.-Ver. 116(4), 201-222 (2014)

33. Mayer, U.F., Simonett, G.: A numerical scheme for axisymmetric solutions of curvature-driven free boundary problems, with applications to the Willmore flow. Interfaces Free Bound. 4(1), 89-109 (2002)

34. Nitsche, J.C.C.: Boundary value problems for variational integrals involving surface curvatures. Quart. Appl. Math. 51(2), 363-387 (1993)

35. Novaga, M., Pozzetta, M.: Connected surfaces with boundary minimizing the Willmore energy. Math. Eng. 2(3), 527-556 (2020)

36. Ou-Yang, Z.C.: Elasticity theory of biomembranes. Thin Solid Films 393, 19-23 (2001)

37. Poisson, S.D.: Mémoire sur les surfaces Élastiques, Mém. de l’Inst., pp. 167-226 (1812; pub. 1816)

38. Pozzetta, M.: On the Plateau-Douglas Problem for the Willmore energy of surfaces with planar boundary curves. ESAIM (European Series in Applied and Industrial Mathematics): COCV (Control, Optimisation and Calculus of Variations), to appear. Available online at https://doi.org/10.1051/cocv/2020049

39. Rivière, T.: Analysis aspects of Willmore surfaces. Invent. Math. 174(1), 1-45 (2008)

40. Rusu, R.E.: An algorithm for the elastic flow of surfaces. Interfaces Free Bound. 7(3), 229-239 (2005)

41. Schätzle, R.: Lower semicontinuity of the Willmore functional for currents. J. Differ. Geom. 81(2), 437456 (2009)

42. Schätzle, R.: The Willmore boundary problem. Calc. Var. Partial Differ. Equ. 37(3-4), 275-302 (2010)

43. Scholtes, S.: Elastic Catenoids. Analysis 31(2), 125-143 (2011)

44. Simon, L.: Existence of surfaces minimizing the Willmore functional. Comm. Anal. Geom. 1(2), 281-326 (1993)

45. Thomsen, G.: Über konforme Geometrie I: Grundlagen der konformen Flächentheorie. Abh. Math. Sem. Hamburg 3, 31-56 (1923)

46. Willmore, T.J.: Note on embedded surfaces. An. Ştiinţ. Univ. Al. I. Cuza Iaşi Seçt. I a Mat 11, 493-496 (1965)

47. Willmore, T.J.: Riemannian Geometry. Oxford Science Publications. The Clarendon Press, Oxford University Press, New York (1993)

Publisher's Note Springer Nature remains neutral with regard to jurisdictional claims in published maps and institutional affiliations. 